\title{
Annular substructures in the transition disks around LkCa 15 and J1610^
}

\author{
S. Facchini ${ }^{1}$, M. Benisty ${ }^{2,3}$, J. Bae ${ }^{4}$, R. Loomis ${ }^{5}$, L. Perez ${ }^{2}$, M. Ansdell ${ }^{6}$, S. Mayama ${ }^{7}$, P. Pinilla ${ }^{8}$, \\ R. Teague ${ }^{9}$, A. Isella ${ }^{10}$, and A. Mann ${ }^{11}$ \\ ${ }^{1}$ European Southern Observatory, Karl-Schwarzschild-Str. 2, 85748 Garching, Germany \\ e-mail: stefano.facchini@eso.org \\ ${ }^{2}$ Unidad Mixta Internacional Franco-Chilena de Astronomía, CNRS, UMI3386, Departamento de Astronomía, Universidad de Chile, \\ Camino El Observatorio 1515, Las Condes, Santiago, Chile \\ ${ }^{3}$ Univ. Grenoble Alpes, CNRS, IPAG, 38000 Grenoble, France \\ ${ }^{4}$ Earth and Planets Laboratory, Carnegie Institution for Science, 5241 Broad Branch Road NW, Washington, DC 20015, USA \\ ${ }^{5}$ NRAO, 520 Edgemont Rd, Charlottesville, VA 22903, USA \\ ${ }^{6}$ Flatiron Institute, Simons Foundation, 162 Fifth Ave, New York, NY 10010, USA \\ 7 The Graduate University for Advanced Studies, SOKENDAI, Shonan Village, Hayama, Miura, Kanagawa 240-0193, Japan \\ ${ }^{8}$ Max Planck Institute for Astronomy, Königstuhl 17, 69117 Heidelberg, Germany \\ ${ }^{9}$ Center for Astrophysics I Harvard \& Smithsonian, 60 Garden Street, Cambridge, MA 02138, USA \\ ${ }^{10}$ Department of Physics and Astronomy, Rice University, 6100 Main Street, MS-108, Houston, TX 77005, USA \\ ${ }^{11}$ Department of Physics and Astronomy, University of North Carolina at Chapel Hill, Chapel Hill, NC 27599, USA
}

Received 26 March 2020 / Accepted 5 May 2020

\begin{abstract}
We present high-resolution millimeter continuum ALMA observations of the disks around the T Tauri stars LkCa 15 and 2MASS J16100501-2132318 (hereafter, J1610). These transition disks host dust-depleted inner regions, which have possibly been carved by massive planets, and they are of prime interest to the study of the imprints of planet-disk interactions. While at moderate angular resolution, they appear as a broad ring surrounding a cavity, the continuum emission resolves into multiple rings at a resolution of $\sim 60 \times 40$ mas ( $~ 7.5 \mathrm{au}$ for $\mathrm{LkCa} 15, \sim 6$ au for $\mathrm{J} 1610)$ and $\sim 7 \mu \mathrm{Jy} \mathrm{beam}^{-1} \mathrm{rms}$ at $1.3 \mathrm{~mm}$. In addition to a broad extended component, LkCa 15 and J1610 host three and two narrow rings, respectively, with two bright rings in LkCa 15 being radially resolved. LkCa 15 possibly hosts another faint ring close to the outer edge of the $\mathrm{mm}$ emission. The rings look marginally optically thick, with peak optical depths of $\sim 0.5$ (neglecting scattering), in agreement with high angular resolution observations of full disks. We performed hydrodynamical simulations with an embedded, sub-Jovian-mass planet and show that the observed multi-ringed substructure can be qualitatively explained as the outcome of the planet-disk interaction. We note, however, that the choice of the disk cooling timescale alone can significantly impact the resulting gas and dust distributions around the planet, leading to different numbers of rings and gaps and different spacings between them. We propose that the massive outer disk regions of transition disks are favorable places for planetesimals, and possibly second-generation planet formation of objects with a lower mass than the planets carving the inner cavity (typically few $M_{\mathrm{Jup}}$ ), and that the annular substructures observed in LkCa 15 and $\mathrm{J} 1610$ may be indicative of planetary core formation within dust-rich pressure traps. Current observations are compatible with other mechanisms contributing to the origin of the observed substructures, in particular with regard to narrow rings generated (or facilitated) at the edge of the $\mathrm{CO}$ and $\mathrm{N}_{2}$ snowlines.
\end{abstract}

Key words. accretion, accretion disks - protoplanetary disks - submillimeter: planetary systems - stars: individual: LkCa 15 stars: individual: J1610

\section{Introduction}

High angular resolution observations of protoplanetary disks show that the existence of substructure in their dust emission is ubiquitous. This is the case in both (sub-)millimeter ( $\mathrm{mm}$ ) emission, largely tracing dust grains with sizes $>100 \mu \mathrm{m}$ (e.g., Andrews 2020), and in scattered light observations at optical and near infrared (NIR) wavelengths, probing sub- $\mu \mathrm{m}$ sized dust grains (e.g., Garufi et al. 2018). The only disks that so far do not show azimuthal or radial substructure in their dust distribution are very compact or faint objects, where the finite

\footnotetext{
* The reduced images are only available at the CDS via anonymous ftp to cdsarc.u-strasbg. fr (130.79.128.5) or via http://cdsarc. u-strasbg.fr/viz-bin/cat/J/A+A/639/A121
}

resolution of the instruments is able to image these disks with just a few (or less) resolution elements across their diameter (e.g., Facchini et al. 2019). Before the advent of the high resolution Atacama Large Millimeter/submillimeter Array (ALMA) and extreme adaptive optics systems on ground based telescopes, photometric surveys had already identified a class of objects with some level of substructure, the so-called transition disks, evidenced from a lack of NIR or mid-IR emission in their spectral energy distribution that indicates a dust-depleted inner region. This was confirmed with sub-mm images that clearly showed the presence of cavities with angular sizes larger than the resolution elements available at the time $\left(0.3^{\prime \prime}, \sim 40-75 \mathrm{au}\right.$; Brown et al. 2009; Andrews et al. 2011) surrounded by a bright and wide ring. Their morphology has been explained as the outcome of planet-disk interactions, with massive companions carving a 
cavity and generating a pressure maximum outside their orbital radius which $\mathrm{mm}$ grains drift towards and accumulate (e.g., Rice et al. 2006). In some cases, the radial extent of the cavities is too large to be explained by a single planet orbiting on a circular orbit and the presence of multiple giant planets was suggested (Dodson-Robinson \& Salyk 2011; Bae et al. 2019). Other physical mechanisms have also been invoked to explain the lack of dust particles in the inner regions of transition disks, such as internal photoevaporation (e.g., Alexander et al. 2006; Gorti \& Hollenbach 2009; Owen et al. 2010) and dead zones (e.g., Regály et al. 2012; Flock et al. 2015).

High angular resolution observations of these objects at $\mathrm{mm}$ wavelengths are crucial to the understanding of the origin of these cavities. The radial and azimuthal brightness distributions of the ring itself can be analyzed as probes of planet-disk interactions, with, for example, asymmetries tracing vortices due to the Rossby Wave instability. Independently of the mechanism clearing the central cavity, it is clear that dust trapping must be occurring in the ring, since $\mathrm{mm}$ dust grains would otherwise naturally drift towards the central star. In these rings, the dust-to-gas ratio is expected to be close to unity due to the efficient trapping at the pressure maximum. In such physical conditions, planetesimal formation is thought to occur efficienty via the streaming-instability (e.g., Youdin \& Goodman 2005; Johansen et al. 2007). A subsequent triggering of core-formation via pebble accretion may occur (e.g., Ormel \& Klahr 2010; Bitsch et al. 2015), even though theoretical models suggest that the growth timescales could be too long for this process to be efficient at large distances from the star within the disk lifetime (e.g. Morbidelli 2020). These rings are likely among the best environments to look for signatures of on-going formation of planetesimals and possibly planetary cores.

Some transition disks have been imaged at high angular resolution at mm wavelengths ( 20-70 mas), showing that the wide rings observed at intermediate resolution are actually composed of smaller-scale features. They show complex morphologies and finer levels of substructure in both radial and azimuthal directions. Multiple rings, spirals, arcs and eccentric features have been detected, showing high level of complexity (e.g., Dong et al. 2018; Andrews et al. 2018; Casassus \& Pérez 2019; Rosotti et al. 2019; Pérez et al. 2019). Interestingly, in the handful of disks that have been observed at similarly high resolution in both scattered light and $\mathrm{mm}$ thermal emission, there is no strict correspondence between the morphological features (e.g., Cazzoletti et al. 2018), indicating that the physical processes responsible for these complex morphologies may have different imprints depending on the disk tracer.

In this paper, we present new ALMA Band $6220 \mathrm{GHz}$ continuum observations of LkCa 15 and 2MASS J161005012132318 (EM*StHA 123, EPIC 204630363, hereafter J1610) at unprecedented angular resolution $(\sim 40 \times 60$ mas, i.e., $\sim 7.5$ and 6 au in radius for LkCa 15 and J1610, respectively). Both objects are classical T Tauri stars hosting transition disks with resolved cavities (Piétu et al. 2006; Ansdell et al. 2020). Interestingly they are also classified as dippers, based on the short term variability seen in their optical light curves (Ansdell et al. 2016; Rodriguez et al. 2017; Alencar et al. 2018). This photometric variability is interpreted as due to a warp in a misaligned inner disk, possibly due to an inclined magnetic field or a companion on an inclined orbit.

LkCa 15 is a well-studied object, which has garnered a lot of attention due to the claims of giant planets in the disk inner cavity (Kraus \& Ireland 2012; Sallum et al. 2015). Recent results show that the emission is however likely due to disk signal
(Thalmann et al. 2016; Mendigutía et al. 2018; Currie et al. 2019). Located at a distance of $158.9 \pm 1.2 \mathrm{pc}$ (Gaia Collaboration 2018), $\mathrm{LkCa} 15$ is a $1.25 \pm 0.10 M_{\odot}$ star, with an age of $\sim 5 \mathrm{Myr}$ and accretion rate of $10^{-9.2 \pm 0.3} M_{\odot} \mathrm{yr}^{-1}$ and a stellar bolometric luminosity of $1.05_{-0.21}^{+0.27} L_{\odot}$ (Donati et al. 2019). We note that previous works used a stellar luminosity of $0.74 L_{\odot}$ derived with the pre-Gaia distance. Dynamical estimates of the central star matches well to the one estimated from the pre-main sequence track (Qi et al. 2019). The disk is very bright in the mm (380 mJy at $870 \mu \mathrm{m}$; Andrews et al. 2011), with a dust disk mass of $\sim 165 M_{\oplus}$ in the optically thin assumption (Isella et al. 2012), and a gaseous disk traced by CO out to $\sim 900$ au (e.g., Jin et al. 2019). While the disk exhibits a clear dust-depleted cavity at mm wavelengths, of $\sim 50$ au in radius (e.g., Piétu et al. 2006; Isella et al. 2012; Pinilla et al. 2018), scattered light observations indicate the presence of an inner disk extending up to $\sim 30 \mathrm{au}$ within the $\mathrm{mm}$ cavity (e.g., Thalmann et al. 2016; Oh et al. 2016). Such a spatial segregation in dust sizes is a natural outcome of planet-disk interactions (Pinilla et al. 2012) and supports the presence of a massive planet orbiting at $\sim 40 \mathrm{au}$.

J1610 is an object that has been studied to a lesser extent. Rizzuto et al. (2015) spectroscopically identified it as a lowmass member of the Upper Sco association. It is located at a distance of $144.7 \pm 2.7 \mathrm{pc}$ (Gaia Collaboration 2018) and the star has a K7.5 spectral type, with evidence of accretion from $\mathrm{H} \alpha$ emission (Ansdell et al. 2016). Using the new Gaia distance, we fitted the SNIFS stellar spectrum and broadband photometric spectral energy distribution (SED) from Ansdell et al. (2016) and obtained a stellar luminosity of $0.46 \pm 0.03 L_{\odot}$, with $T_{\text {eff }}=3950 \pm 80 \mathrm{~K}$ (more details in Appendix A). By using the Siess et al. (2000) pre-main sequence evolutionary tracks as done by Barenfeld et al. (2016) for a large sample of Class II objects in Upper-Scorpius, we obtain a stellar mass of $0.67 \pm 0.11 M_{\odot}$, where we assumed a metallicity $Z=0.02$ and no convective overshoot. The disk was detected with the Sub-millimeter Array (SMA) with an integrated flux density of $28.0 \pm 1.5 \mathrm{mJy}$ at $1.3 \mathrm{~mm}$ (Ansdell et al. 2016), with estimated dust mass of $15 M_{\oplus}$ in the optically thin and isothermal assumptions, and has been shown to have a cavity of $\sim 20 \mathrm{au}$ in radius from previous moderate resolution ALMA observations (Ansdell et al. 2020).

The paper is organized as follows. In Sect. 2 the observations, data calibration and imaging parameters are presented. Section 3 summarizes the data analysis, and Sect. 4 compares the data with hydrodynamical simulations of planet-disk interaction. In Sect. 5, we discuss our results and present our conclusions in Sect. 6.

\section{ALMA observations and data reduction}

LkCa 15 and J1610 were observed in Band 6 as part of the ALMA Program \#2018.1.01255.S. The observations were carried out with different configurations in order to provide good uvcoverage at different spatial frequencies, using nominal C43-5, C43-8 and C43-9 configurations for $\mathrm{LkCa} 15$, and C43-6 and C43-8 for J1610. Details of the observations are reported in Table 1. The maximum recoverable scale for $\mathrm{LkCa} 15$ and J1610 is $3.7^{\prime \prime}$ and $1.8^{\prime \prime}$, respectively. The spectral setup had four spectral windows, three dedicated for continuum observations in TDM mode, and one dedicated for high spectral resolution observations of the ${ }^{12} \mathrm{CO}$ line. In this paper we focus on the three continuum spectral windows only, which have central rest frequency of $214,216.2$ and $229 \mathrm{GHz}$. 
Table 1. Log of ALMA observations used in this paper.

\begin{tabular}{|c|c|c|c|c|c|c|c|c|}
\hline Object & Date & Antennas & Min. baseline & Max. baseline & Time on source & Bandpass calibrator & Phase calibrator & Flux calibrator \\
\hline $\mathrm{LkCa} 15$ & 18.11.2018 & 45 & $15 \mathrm{~m}$ & 1397 m & $31 \mathrm{~min}$ & $\mathrm{~J} 0510+1800$ & $\mathrm{~J} 0426+2327$ & $\mathrm{~J} 0510+1800$ \\
\hline $\mathrm{LkCa} 15$ & 13.07.2019 & 40 & $111 \mathrm{~m}$ & $12644 \mathrm{~m}$ & $37 \mathrm{~min}$ & $\mathrm{~J} 0510+1800$ & $\mathrm{~J} 0431+2037$ & $\mathrm{~J} 0510+1800$ \\
\hline LkCa 15 & 19.07.2019 & 43 & $96 \mathrm{~m}$ & $8547 \mathrm{~m}$ & $37 \mathrm{~min}$ & $\mathrm{~J} 0510+1800$ & $\mathrm{~J} 0431+2037$ & $\mathrm{~J} 0510+1800$ \\
\hline $\mathrm{J} 1610$ & 03.09 .2019 & 46 & $38 \mathrm{~m}$ & $3143 \mathrm{~m}$ & $19 \mathrm{~min}$ & $\mathrm{~J} 1517-2422$ & J1551-1755 & J1517-2422 \\
\hline $\mathrm{J} 1610$ & 17.07.2019 & 43 & $92 \mathrm{~m}$ & $8547 \mathrm{~m}$ & $43 \mathrm{~min}$ & J1427-4206 & J1551-1755 & J1427-4206 \\
\hline $\mathrm{J} 1610$ & 18.07.2019 & 46 & $92 \mathrm{~m}$ & $8547 \mathrm{~m}$ & $19 \mathrm{~min}$ & J1427-4206 & J1551-1755 & J1427-4206 \\
\hline
\end{tabular}
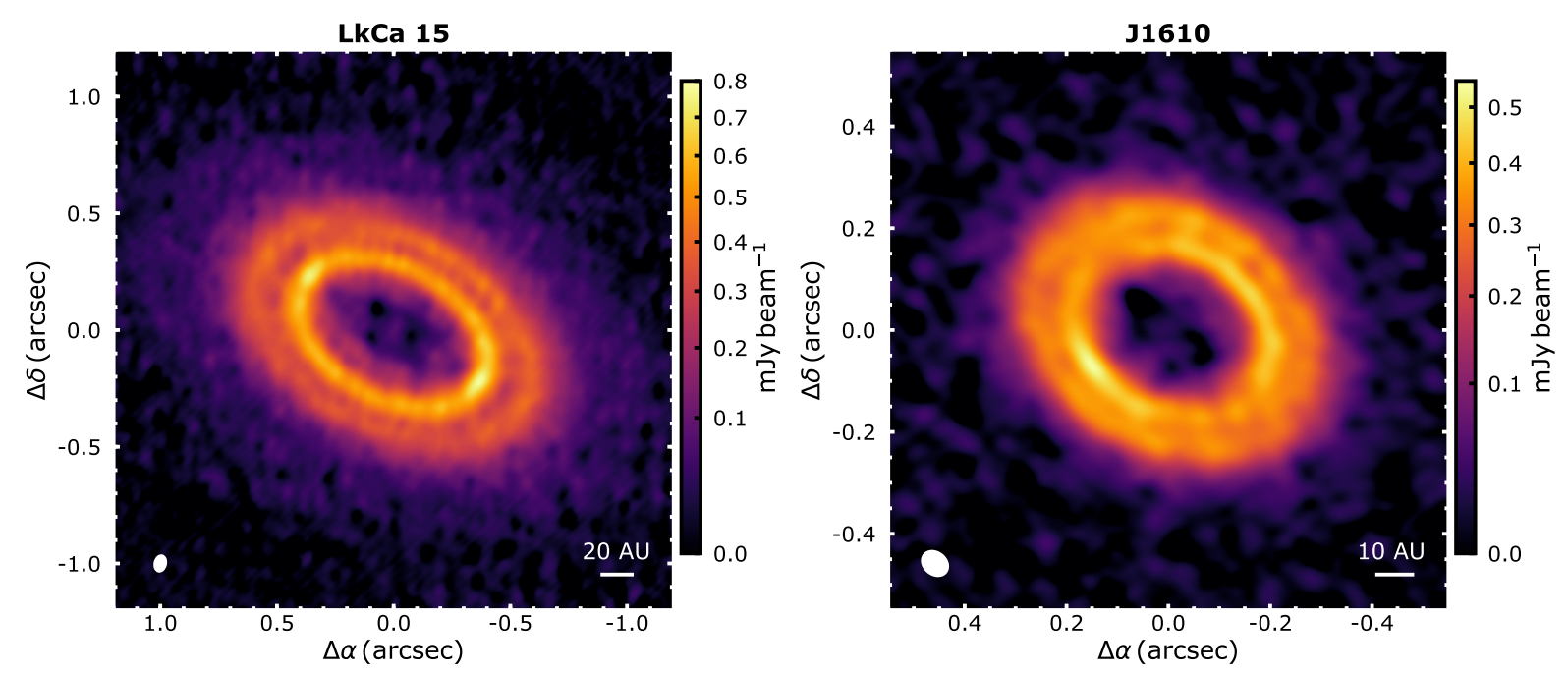

Fig. 1. Intensity maps of LkCa 15 (left panel) and J1610 (right panel). The two panels have different angular scales. The color scale has been stretched to highlight the fainter regions of the disks.

The data were calibrated using the CASA package, version 5.6 (McMullin et al. 2007). Self-calibration was performed on all data-sets, leading to a good improvement in the signal-tonoise ratio $(\mathrm{S} / \mathrm{N})$. The visibilities were merged using the concat task in CASA, and spectrally re-binned on $250 \mathrm{MHz}$ channels to avoid bandwidth smearing. Images in the sky plane were produced using the tclean task, with the multiscale cleaning algorithm allowing for point source emission. Elliptical masks were applied, with position angle and inclination derived from $(u, v)$-plane analysis (see Sect. 3.2), and a semi-major axis of 1.7" and $1^{\prime \prime}$ for LkCa 15 and J1610, respectively. The de-convolution was performed down to a cleaning threshold of $1 \sigma$, which maximises the flux in the clean model. The residuals in the final images were rescaled by the ratio of the clean beam and dirty beam (Jorsater \& van Moorsel 1995), which reduced the rms noise level by $\sim 50 \%$. This is to correct for the fact that the final image is the sum of the restored clean components (in units of clean beams) and of the residuals (in units of dirty beams); to properly estimate the flux of the residuals, a re-normalization factor equal to the ratio of the two beam areas is applied to them. This method has been successfully applied in Very Large Array (VLA) surveys such as THINGS (Walter et al. 2008), and more recently by Pinte et al. (2020) on ALMA data of protoplanetary disks. Different weighting schemes were tested to produce the images. The best compromise between angular resolution and $\mathrm{S} / \mathrm{N}$ for $\mathrm{LkCa} 15$ is with a Briggs robust weighting of 0 , whereas for J1610 we opted for a Briggs robust weighting of 0.3 . The resulting synthesized beam for LkCa 15 is $68 \times 47$ mas, with a position angle (P.A.) of $347.4^{\circ}$. The rms noise level is $\sim 6.9 \mu \mathrm{Jy}_{\text {beam }}^{-1}$, as estimated from an annulus centered in the phase center with a $2.5-4$ " range in radii, and the image has a $\mathrm{S} / \mathrm{N}$ of $\sim 115$ at the peak. For J1610, the synthesized beam is $55 \times 43$ mas with a PA of $50.3^{\circ}$, and the rms noise level is $\sim 7.2 \mu \mathrm{Jy}_{\text {beam }}{ }^{-1}$. The $\mathrm{S} / \mathrm{N}$ at the peak is $\sim 75$. The recovered flux density within the cleaning mask is $136.4 \pm 0.1$ and $30.8 \pm 0.1 \mathrm{mJy}$ for LkCa 15 and J1610, respectively. For both disks, the integrated flux densities are in agreement with previous observations at the same wavelength with different interferometers within the $10 \%$ calibration uncertainties, in particular the IRAM PdBI array and CARMA for LkCa 15 (Piétu et al. 2006; Isella et al. 2012) and SMA for J1610 (Ansdell et al. 2016). The resulting images are shown in Fig. 1.

\section{Results}

\subsection{Images}

The intensity maps reported in Fig. 1 clearly show that the two transition disks have substructure, with the bright $\mathrm{mm}$ ring observed in earlier observations separating into distinct multiple rings. Two clear rings are easily observable by looking at the images, and they look remarkably similar in the two disks. One major difference is that the rings observed in J1610 would lay within the cavity of $\mathrm{LkCa} 15$, due to their different angular scales. Compared to other transition disks observed at high angular resolution, these two disks do not show high level of asymmetry, lacking prominent arcs and spirals features as observed for example in massive disks around Herbig stars as HD 135344B (Cazzoletti et al. 2018) and MWC 758 (Dong et al. 2018). However, both disks present azimuthal asymmetries along 
Table 2. Median of the marginalized posteriors of the fitted parameters for LkCa 15, with associated statistical uncertainties from the 16th and 84th percentiles of the marginalized distributions.

\begin{tabular}{lccccc}
\hline \hline Disk geometry & \multicolumn{5}{l}{} \\
\hline$i$ & $\left({ }^{\circ}\right)$ & $50.16 \pm 0.03$ & & & \\
PA & $\left({ }^{\circ}\right)$ & $61.92 \pm 0.04$ & & & \\
$\Delta$ RA & $(\mathrm{mas})$ & $3.6 \pm 0.2$ & & & \\
$\Delta$ Dec & $(\mathrm{mas})$ & $-6.0 \pm 0.2$ & & & \\
\hline Ring parameters & & $\mathrm{B} 47$ & $\mathrm{~B} 69$ & $\mathrm{~B} 100$ & Broad component \\
\hline$I_{i}$ & $(\log$ Jy steradian & \\
$R_{i}$ & $9.078_{-0.019}^{+0.018}$ & $9.866_{-0.005}^{+0.004}$ & $9.535_{-0.003}^{+0.003}$ & $9.029_{-0.010}^{+0.009}$ \\
$R_{i}$ & $(\mathrm{au})$ & $47.33_{-1.20}^{+1.53}$ & $68.99_{-0.15}^{+0.17}$ & $100.11_{-0.19}^{+0.19}$ & $88.92_{-1.37}^{+1.53}$ \\
$\sigma_{i}$ & $(\mathrm{au})$ & $9.33_{-1.11}^{+1.28}$ & $6.32_{-0.16}^{+0.16}$ & $14.51_{-0.21}^{+0.21}$ & $59.87_{-0.57}^{+0.54}$ \\
\hline
\end{tabular}

Notes. The center of the emission is computed with respect to the phase center of the visibilities, i.e., RA $(\mathrm{J} 2000)=04: 39: 17.8059$ and Dec $(\mathrm{J} 2000)=$ $+22: 21: 03.048$.

Table 3. Same as Table 2 for $\mathrm{J} 1610$ and using RA $(\mathrm{J} 2000)=16: 10: 05.0032$ and Dec $(\mathrm{J} 2000)=-21: 32: 32.378$.

\begin{tabular}{lcccc}
\hline \hline Disk geometry & & & \\
\hline$i$ & $\left({ }^{\circ}\right)$ & $37.80 \pm 0.20$ & & \\
PA & $\left({ }^{\circ}\right)$ & $60.51 \pm 0.34$ & & \\
$\Delta$ RA & $($ mas $)$ & $-0.9 \pm 0.4$ & & \\
$\Delta$ Dec & $($ mas $)$ & $-1.8 \pm 0.3$ & & \\
\hline Ring parameters & & $\mathrm{B} 29$ & $\mathrm{~B} 41$ & Broad component \\
\hline$I_{i}$ & $(\log$ Jy steradian & & \\
$R_{i}$ & $(\mathrm{au})$ & $9.925_{-0.019}^{+0.019}$ & $9.714_{-0.019}^{+0.017}$ & $9.225_{-0.067}^{+0.064}$ \\
$\sigma_{i}$ & $(\mathrm{au})$ & $28.79_{-0.10}^{+0.10}$ & $41.11_{-0.15}^{+0.13}$ & $33.81_{-0.61}^{+0.50}$ \\
\hline
\end{tabular}

the north-west - south-east direction, and they are particularly evident in $\mathrm{J} 1610$.

Radial profiles. In order to analyze the intensity profile, the intensity maps were re-centered, deprojected, and azimuthally averaged using the geometrical parameters reported in Tables 2 and 3 and determined from fitting the visibilities. Details of the geometrical parameters determination can be found in Sect. 3.2. Radial bins of 9 mas are used, and errors are computed as the quadratic sum of the standard deviation of the intensity across pixels in each radial bin and the rms of the observations divided by the square root of the number of independent beams sampling the same radial bin in the azimuthal direction.

The resulting intensity profiles are reported in Fig. 2 and show radial substructure, with two narrow rings emerging in both LkCa 15 and J1610, and an underlying extended broad component. A figure with the same profiles in logarithmic scale highlights the faint extended emission (Fig. B.1). The intensity peak of the outer ring is always fainter than the inner ring peak, which can be explained by a drop in dust temperature or optical depth. On average, the outer ring is brighter in the NW side of the disk by $\sim 10 \%$ (Fig. 2, right) with the NW side being the close side of the disk to the observer (Thalmann et al. 2015). This same effect seems to be even more pronounced in lower resolution band 9 data (van der Marel et al. 2015), and may be indicative of azimuthally asymmetric illumination of the disk due to shadowing (Thalmann et al. 2016).

In LkCa 15, the inner ring shows a "shoulder" extending inwards in the cavity, similarly to other transition disks imaged at high angular resolution (e.g., Pérez et al. 2019; Huang et al. 2020). The shoulder might indicate the presence of a ring unresolved at the spatial resolution of the observations. Interestingly, this structure is slightly more prominent in the south-east side of the disk, as shown in Fig. 2, right, suggesting that it is azimuthally asymmetric. Other disks have shown asymmetric structure reminiscent of streamers or filaments trailing a major symmetric dust ring (Isella et al. 2018; Pérez et al. 2019), but the spatial resolution of the observation of $\mathrm{LkCa} 15$ impedes a similar characterization of the morphological feature. Both disks show a shallower tail in the outer regions, which has been already observed in LkCa 15 and other transition disks (e.g., Pinilla et al. 2018; Jin et al. 2019), in agreement with radial drift models predicting a smaller maximum grain sizes in the outer regions of the disk, leading to lower opacities at $\mathrm{mm}$ wavelength. In both cases, this extended emission shows a shoulder (meaning an intensity profile with negative second derivative), which may be tracing a shallow ringed structure at large radii. These outer shoulders are located at $\sim 175$ and at $\sim 63$ au in $\mathrm{LkCa} 15$ and J1610, respectively.

Emission within the cavity. The cavities of both disks present low levels of emission (see Figs. 2 and B.1). In the following, we refer to an uncertainty driven by the nominal rms given in Sect. 2, and estimated in the outer regions of the maps. We note that it might underestimate the rms in the inner regions, for which deconvolution errors are significant due to the high dynamic range. J1610 does not show clear centrally peaked emission, with an average surface brightness within the cavity of $\sim 2 \sigma$. The LkCa 15 disk also shows a low surface brightness distributed within the whole cavity, but with clear inner disk unresolved emission located at the center, with a peak intensity of $73.7 \pm 6.9 \mu \mathrm{Jy} \mathrm{beam}^{-1}$, reaching a $S / N \sim 10.7 \sigma$. The total 

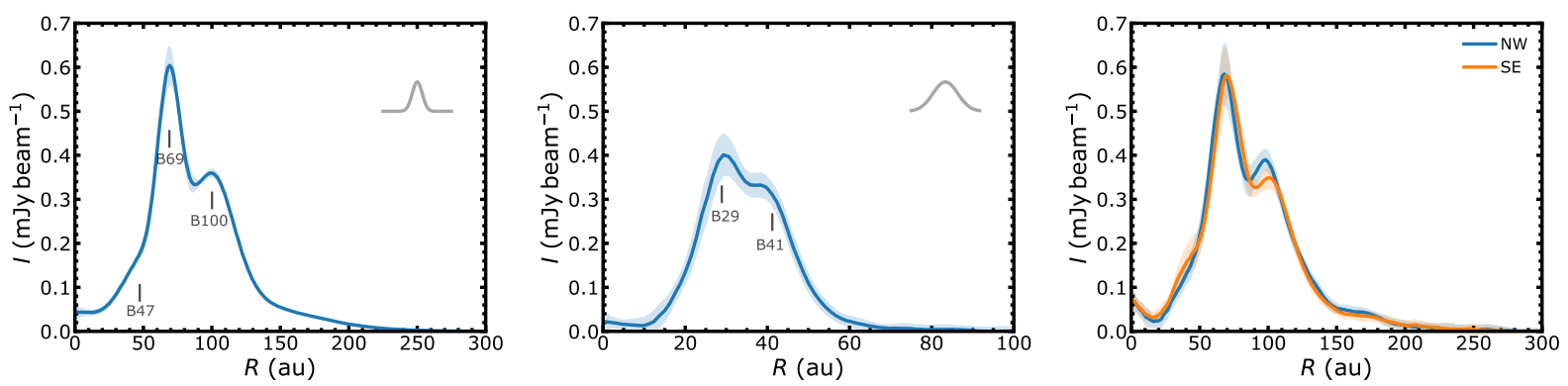

Fig. 2. Left and middle: deprojected and azimuthally averaged radial intensity profile of the continuum emission of LkCa 15 (left) and $\mathrm{J} 1610$ (middle). The ribbon shows the quadratic sum of the standard deviation of the intensity across pixels in each radial bin and the rms of the observations divided by the square root of the number of independent beams sampling the same radial bin in the azimuthal direction. The Gaussian profile in the top right corner of the panels shows the FWHM of the average of major and minor axis of the synthesized beam. Right: azimuthal average of the south-east, north-west sides of $\mathrm{LkCa} 15$, respectively, where the disk has been divided in two sub-regions along the major axis. The inner shoulder is more prominent in the south-east side of the disk.

flux within an area slightly larger than one beam (with same PA, and major and minor axes 1.2 larger than the ones of the beam) leads to a flux density of $58.9 \pm 7.2 \mu \mathrm{Jy}$. Isella et al. (2014) also detected a point source at the center of LkCa 15 with VLA observations at $7 \mathrm{~mm}$ with 70 mas resolution, with a flux density of $16.6 \pm 3.6 \mu \mathrm{Jy}$. Taking into account a $10 \%$ uncertainty in the flux calibration of both the VLA and the ALMA observations presented in this paper, and assuming that the errors are Gaussian, we obtain a spectral index of $0.9 \pm 0.2$ between 1.35 and $7 \mathrm{~mm}$. This low value is incompatible with emission due to dust thermal radiation alone, and suggests that at $7 \mathrm{~mm}$ there may be a contribution from either free-free or synchrotron emission by the ionized gas in the proximity of the central star (Panagia \& Felli 1975; Reynolds 1986).

The LkCa 15 image additionally presents a localized, unresolved, bright emission within the cavity, at a radius of $\sim 0.22^{\prime \prime}$ ( $\sim 35 \mathrm{au}$ ) and along a position angle of $\sim 236^{\circ}$ (see Fig. D.1, left). The overbrightness is seen when imaging the disk with different weightings, in particular with robust parameter $<0.3$ that allows to separate it from the inner bright $\mathrm{mm}$ ring. To verify whether it is an imaging artifact, we measure the peak intensity at the location of the feature when imaging the disk with different weighting schemes, in particular with Briggs weighting and robust parameter ranging from 0.5 to -0.5 . The results are shown in Fig. D.1, right panel. The peak intensity varies with imaging parameters, ranging between $112 \pm 21$ and $211 \pm 14 \mu \mathrm{Jy} \mathrm{beam}^{-1}$ (for robust parameter -0.5 and 0.3 , leading to a nominal $\mathrm{S} / \mathrm{N}$ of $\sim 5.3$ and $\sim 15 \sigma$, respectively). This suggests that the feature is likely an imaging artifact, since the peak intensity of an unresolved point source should not vary with resolution element. However, considering the proximity of the over-intensity to the bright $\mathrm{mm}$ ring, we expect the peak intensity to decrease with increased angular resolution as they are better separated. We discuss this further in the $u v$-plane analysis in Sect. 3.2. This discussion highlights the challenges of detecting faint point sources within cavities of transition disks, due to the high dynamic range and image reconstruction artifacts. Deeper observations with different array configurations and possibly different frequencies are needed to reach a final conclusion about the nature of these faint features, which can be interpreted as circumplanetary disks. Even more convincing cases, as the one of PDS 70c (Isella et al. 2019), still need confirmation with new observations.

\subsection{Modeling of the continuum emission}

In order to better characterize the continuum emission described in Sect. 3.1, we model the intensity maps in the $(u, v)$-plane. We describe the continuum brightness distribution of LkCa 15 and $\mathrm{J} 1610$ by fitting the visibility points using an axisymmetric parametric model that consists in a set of radially Gaussian rings. The brightness profile of each model follows:

$I(R)=\sum_{i=1}^{N} I_{i} \mathrm{e}^{-\left(R-R_{i}\right)^{2} / 2 \sigma_{i}^{2}}$

where $N$ is the number of rings considered, $I_{i}$ is the peak intensity at radius $R_{i}, \sigma_{i}$ is the ring width. As each ring has three free parameters $\left(I_{i}, R_{i}\right.$ and $\left.\sigma_{i}\right)$, the model has $3 \times N$ free parameters, plus four global disk parameters that are fitted for all rings together: the disk inclination $(i)$, position angle (PA) and offset from the phase center $(\triangle \mathrm{RA}, \Delta \mathrm{Dec})$. Inspired by the image analysis (Sect. 3.1), the model consists in a broad ring and three or two narrow rings for $\mathrm{LkCa} 15$ and J1610, respectively, and is described with 16 and 13 free parameters, respectively. Flat priors are used for all parameters, with the constraint that one of the Gaussian rings has $\sigma_{i}>0.15^{\prime \prime}$, in order to reproduce the broad component at large radii. For each set of parameters, the Fourier transform of the model image is computed and sampled in the $(u, v)$ points of the dataset using GALARIO (Tazzari et al. 2018). We sample the posterior distribution of our parameters with the emcee package (Foreman-Mackey et al. 2013) with 160 walkers over 10000 steps after 2000 steps of burn-in.

Tables 2 and 3 report the parameters of the maximum likelihood model for both targets, as well as statistical uncertainties for each parameter estimated from the 16th and 84th percentiles of the marginalized distributions. The error estimates have been computed after thinning the chains to avoid dependant samples in the posterior distributions. The brightness distribution of LkCa 15 is well represented by 3 narrow Gaussian rings centered at $\sim 47, \sim 69$ and $\sim 100$ au (with widths between $\sim 6$ and $\sim 14 \mathrm{au}$ ) and a broad component centered at $\sim 89$ au with a width of $\sim 60 \mathrm{au}$. In J1610, a similar morphology leads to a good fit, with two narrow $\sim 2-3$ au-wide Gaussian rings located at $\sim 29$ and $\sim 41$ au and a broad component with a width of $\sim 15 \mathrm{au}$. The similar width of the two Gaussian rings in J1610 suggests that the estimated width of the rings is limited by the angular resolution of the observations (the Full Width Half Maximum - FWHM of the beam along the minor axis is $\sim 2 \sigma_{i}$ ). The Gaussian curves composing the best-fit models are shown as dashed lines in Fig. 3.

Figure 4 provides the images of the data, model and residuals for the best-fit model, synthesized with the same imaging parameters. The comparison between the observed visibilities and the model as a function of $(u, v)$ distance are shown in 

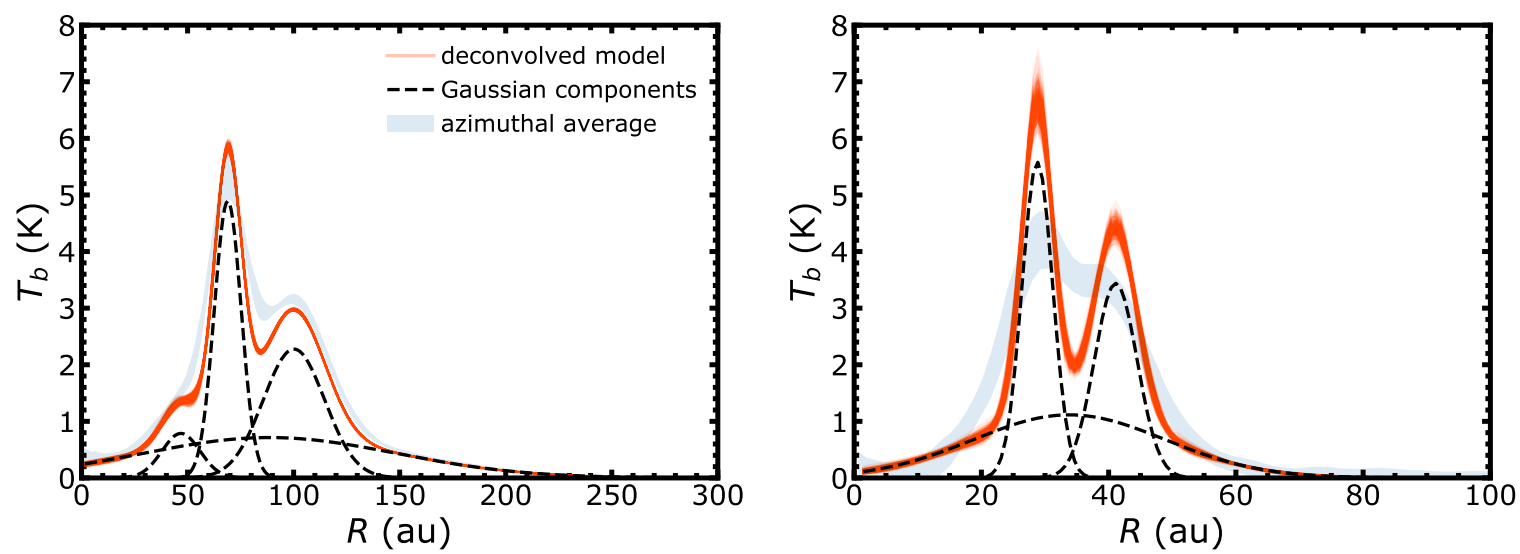

Fig. 3. Deconvolved brightness temperature radial profiles of LkCa 15 (left panel) and J1610 (right panel), calculated in the Rayleigh-Jeans approximation (see Sect. 3.2). The red curves show 800 random realizations of the posterior distribution of the GALARIO model, while the dashed black lines indicate the best fit of the Gaussian components used in the model. The underlying blue shaded region shows the deprojected and azimuthally averaged brightness temperature profile derived from Fig. 1 (see Sect. 3.1).
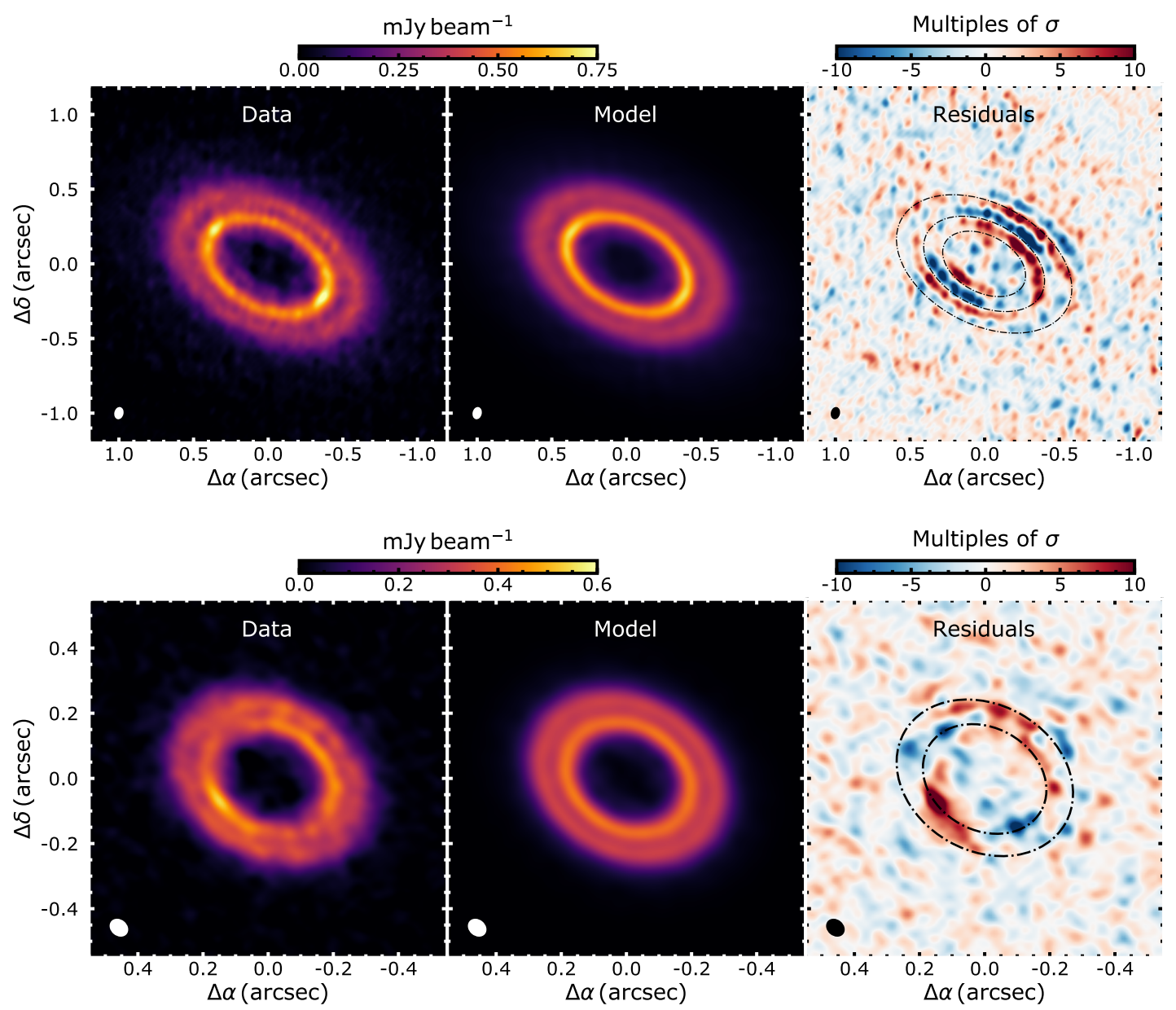

Fig. 4. From left to right: intensity map of the data, best fit model, and residual visibilities for LkCa 15 (top) and J1610 (bottom). The intensity map of the best fit model and residuals have been generated using the same $u v$-sampling as the original data. In the residual map, the black dashed-dotted lines show the location of the rings (see Tables 2 and 3).

Fig. B.2. While the models reproduce well the overall morphology of the emission for both targets, residuals are found at high spatial frequencies. In $\mathrm{LkCa} 15$, at the $10 \sigma$ level, residuals are found slightly inwards and outwards of the rings indicating a more complex morphology than prescribed with a Gaussian. The width of the rings in $\mathrm{LkCa} 15$ is larger than the resolution element, indicating that the rings are partly resolved and deviations from a Gaussian profile can be detected with these observations. Interestingly, both negative and positive residuals are mostly seen along the minor axis, which indicates that the intensity prescription is a good representation of the data where the linear resolution is maximum (along the major axis). The residuals along the minor axis can be explained by projection and radiative transfer effects with a finite ring vertical 
thickness (see also discussion in Huang et al. 2020). The NW over-brightness in both B69 and B100 is apparent in the residuals, as is the azimuthal asymmetry in the inner shoulder (B47). This confirms the analysis in the image plane, with the shoulder being more prominent in the SE side of the disk (Fig. 2, right). In $\mathrm{J} 1610$, the largest residual is azimuthally broad and located in the SE. Interestingly, the positive and negative residuals are mostly located along almost perpendicular directions (minor and major axis, respectively) which could be related to shadowing by the inner disk, as seen in DoAr 44 (Casassus et al. 2018). In both disks, the outer shoulder at $\sim 175$ and $\sim 63$ au is not seen in the residuals (nor in their azimuthal average), with the extended Gaussian component reproducing the intensity profile within $3 \sigma$. Deeper observations might be needed to test whether they can be attributed to shallow rings at a large distance from the star.

Within the central cavities, no residuals are found above the $4 \sigma$ level. The models shown in Fig. 3 both present low surface brightness emission within the cavities. Such prescription works well in both cases, even though the $\mathrm{S} / \mathrm{N}$ within the cavities is low enough in both disks that other functional forms could have been equally representative of the intensity distribution. In $\mathrm{LkCa} 15$, the point like feature discussed in Sect. 3.1 is not recovered in the residuals above $4 \sigma$, suggesting again that this is due to an imaging artifact driven by the high dynamic range of the observations.

\section{Comparison with hydrodynamical simulations}

In this section, we aim to study the conditions for the formation of multiple rings in transition disks that host an inner dustdepleted cavity. We assume that the rings are caused by the interaction with a planet located at the pressure maximum at the edge of the central cavity. We use the better resolved LkCa 15 as a test case. We stress that our goal is not to reproduce a perfect match with the observations, since this would not imply that the solution is unique and would require fine-tuning of the hydrodynamical simulations while lacking possibly relevant physical mechanisms.

\subsection{Set-up}

We adopt a parameterized initial gas surface density profile based on the analysis of low-resolution ${ }^{12} \mathrm{CO}(J=3-2)$ observations of LkCa 15 by Jin et al. (2019), which well reproduces the inner cavity imaged at lower angular resolution. The profile we use follows

$\Sigma_{\mathrm{g}, \text { init }}(R)=\Sigma_{c}\left(\frac{R}{R_{c}}\right)^{-\eta} \arctan \left[\left(\frac{R}{R_{c}}\right)^{\psi}\right]$,

where $R_{c}=65 \mathrm{au}, \eta=4$, and $\psi=10$. We choose $\Sigma_{c}=41 \mathrm{~g} \mathrm{~cm}^{-2}$ so that the total gas disk mass inside of 600 au is $0.1 M_{\odot}$. We point out that our choice of $R_{c}=65$ au differs from the bestfit value of Jin et al. (2019), $R_{c}=45$ au. We need to adopt $R_{c}=65$ au because grains are aerodynamically dragged towards the pressure peak near $R=R_{c}$ and form a ring there; with $R_{c}=45$ au, hydrodynamical simulations show that a ring forms at a too-small radius compared with the continuum observation.

Using the surface density profile described as in Eqs. (2), we create the initial disk temperature by running iterative Monte Carlo radiative transfer (MCRT) calculations using RADMC3D (Dullemond et al. 2012), following the method described in Appendix A of Bae et al. (2019). To briefly summarize, this iteration finds self-consistent three-dimensional density and stellar irradiation-dominated temperature structures that satisfy the hydrostatic equilibrium. We assume $10^{-3} M_{\odot}$ of total dust mass distributed among grains with sizes between $0.01 \mu \mathrm{m}$ and $1 \mathrm{~cm}$, adopting a power-law size distribution with power-law exponent of -3.5 . We further assume that small dust grains having sizes between 0.01 and $1 \mu \mathrm{m}$ are perfectly coupled with disk gas and determine the disk temperature profile. This small grain component has total $9 \times 10^{-6} M_{\odot}$ with the assumed power-law size distribution. For the small grain composition, we assume that they are compact monomers consist of $60 \%$ silicate and $40 \%$ amorphous carbon, having an internal density of $2.7 \mathrm{~g} \mathrm{~cm}^{-3}$. We adopt optical constants of silicate and amorphous carbon from Draine \& Lee (1984) and Li \& Greenberg (1997), respectively. We assume a stellar radius of $1.6 R_{\odot}$ and an effective temperature of $4500 \mathrm{~K}$ (Donati et al. 2019) for the MCRT iterations. Each iteration runs with $10^{9}$ photon packages.

The resulting density-weighted, vertically integrated temperature from the MCRT iterations is presented in Fig. C.1. We note that the disk temperature drops rapidly around the location of the density peak at $65 \mathrm{au}$ as stellar photons are scattered/absorbed at the wall outside of the inner cavity. Adopting a stellar mass of $1.25 M_{\odot}$ (Donati et al. 2019) and a mean molecular weight of 2.4 , the disk aspect ratio $H / R$ between 30 and 300 au can be well described by the following functional form:

$\frac{H}{R}=0.082\left(\frac{R}{R_{c}}\right)^{0.375}+0.022\left[1+\tanh \left(-\frac{R-50 \mathrm{au}}{15 \mathrm{au}}\right)\right]$.

Using the surface density and temperature profiles described in Eqs. (2) and (3), we obtain initial azimuthal velocity that satisfies hydrostatic equilibrium. The initial radial velocity is set to zero.

\subsection{Hydrodynamical simulations}

We carry out two-dimensional hydrodynamical simulations using the Dusty FARGO-ADSG code (Baruteau et al. 2019). This is an extended version of the publicly available FARGO-ADSG (Masset 2000; Baruteau \& Masset 2008a,b), with Lagrangian test particles implemented (Baruteau \& Zhu 2016). The simulation domain extends from 20 to 300 au in the radial direction and covers the entire $2 \pi$ in azimuth. We adopt 672 logarithmicallyspaced grid cells in the radial direction and 1556 uniformlyspaced grid cells in the azimuthal direction. With this choice, one scale height at the location of the planet is resolved with 22 grid cells in both radial and azimuthal directions. A wave-damping zone (de Val-Borro et al. 2006) is implemented at both inner and outer radial boundaries. The disk viscosity $v$ is characterized by a uniform viscosity parameter $\alpha=10^{-4}$, where $v=\alpha c_{\mathrm{s}} H$, with $c_{\mathrm{s}}$ being the local sound speed.

We insert a 60 Earth-mass planet at $70 \mathrm{au}$, at the radial location of the middle continuum ring in the $\mathrm{LkCa} 15$ disk, assuming a fixed circular orbit. This planetary mass has been chosen after running a coarse grid of different planet masses: 30,60 , and $90 M_{\oplus}$. In short, with the viscosity considered in the model, a $30 M_{\oplus}$ planet does not create any rings and gaps, whereas a $90 M_{\oplus}$ planet creates a qualitatively similar morphology to a $60 M_{\oplus}$ planet in terms of the number of rings and gaps, while the contrast between rings and gaps is much larger. A more general discussion about the model degeneracy can be found in Sect. 5. We note that we take an agnostic approach about the origin of the inner cavity and we do not introduce additional planets in the inner cavity, since this would introduce a high number of additional free parameters. 

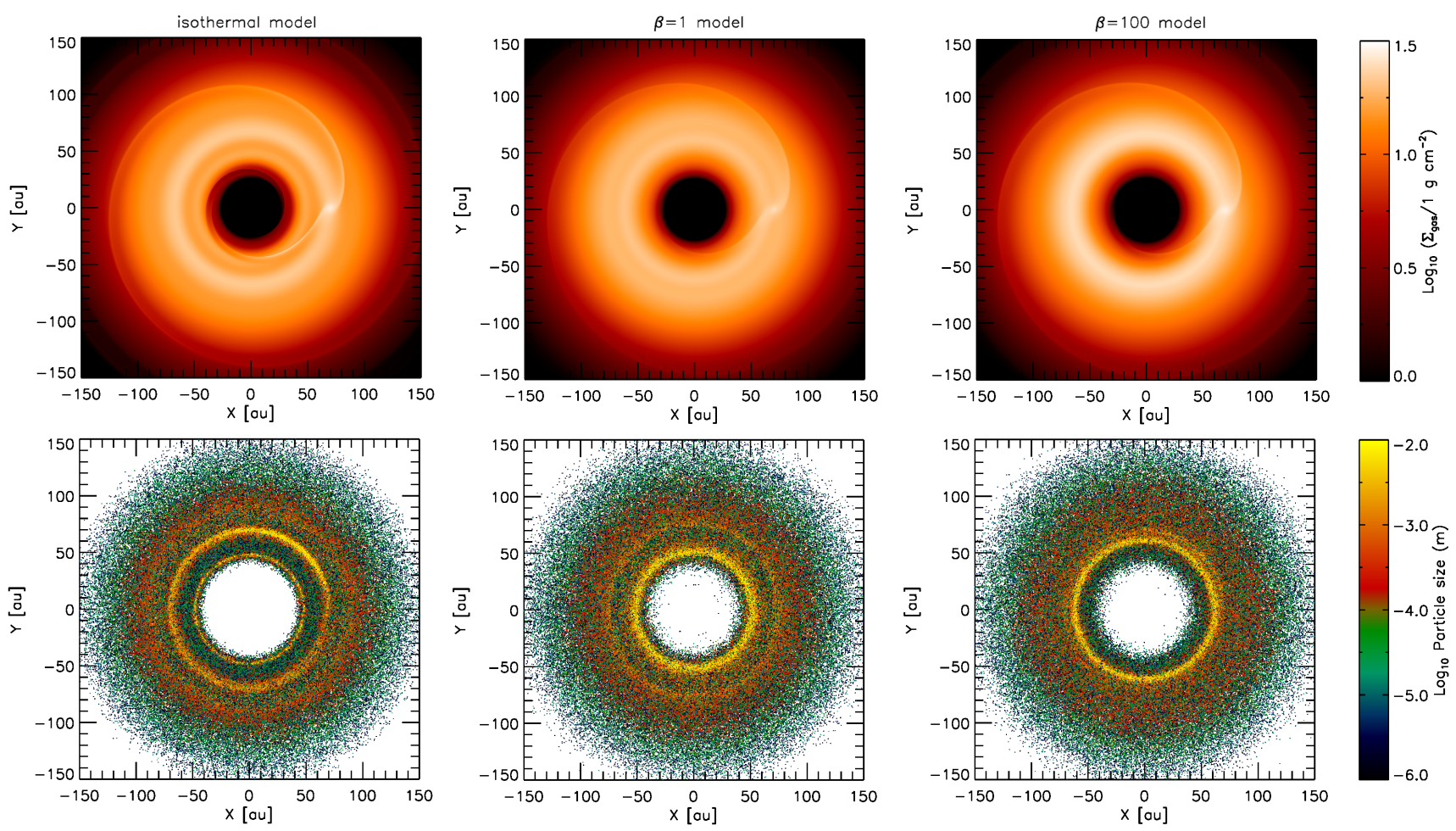

Fig. 5. Two-dimensional distributions of (upper panels) gas and (lower panels) particles. From left to right: (left) isothermal model, (middle) $\beta=1$ model, and (right) $\beta=100$ model. The planet is located at $(X, Y)=(70 \mathrm{au}, 0 \mathrm{au})$. The disk and planet orbit clockwise about the central star.
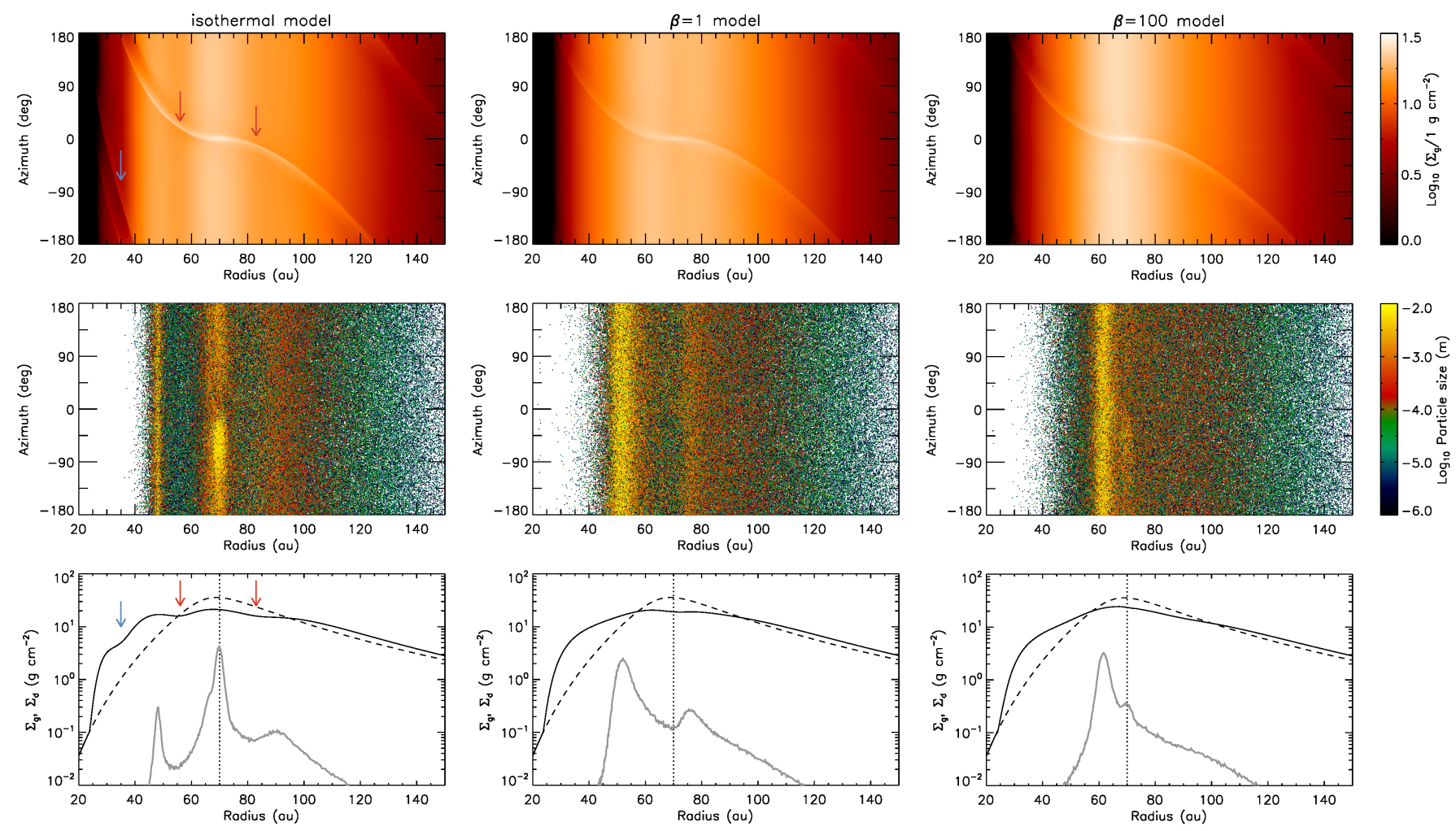

Fig. 6. Two-dimensional distributions of (upper panels) gas and (middle panels) particles in a radius-azimuthal angle plane. Lower panels: azimuthally-averaged radial profiles of gas (solid black lines) and dust (solid gray lines) surface density. The black dashed curves present the initial gas surface density profile, while the black dotted lines present the radial location of the planet. In the isothermal model, the red and blue arrows indicate the location of the shocks due to the primary (red) and secondary (blue) spiral arms. From left to right: (left) isothermal model, (middle) $\beta=1$ model, and (right) $\beta=100$ model. The planet is located at $(R, \phi)=\left(70 \mathrm{au}, 0^{\circ}\right)$. The disk rotation is upward in the upper and middle panels. 


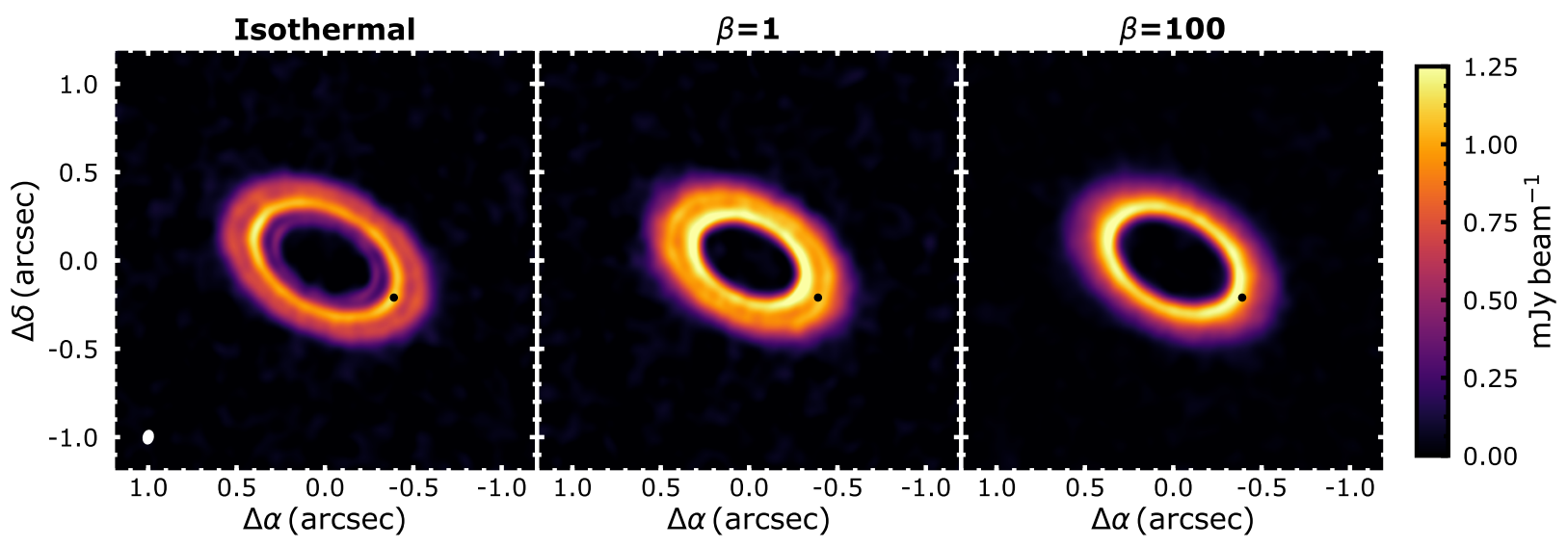

Fig. 7. Simulated continuum images based on (left) isothermal, (middle) $\beta=1$, and (right) $\beta=100$ models. The location of the planet $\left(-0.39^{\prime \prime},-0.21^{\prime \prime}\right)$ is shown with a black filled circle in each panel. The synthesized beam is shown with a white ellipse in the lower-left corner of the left panel. We note the diverse continuum morphology that various treatments of the disk thermodynamics can produce.

Simulations are run for 1000 planetary orbits $(\simeq 0.52 \mathrm{Myr})$, by which time the gas and particle distribution reached a quasisteady state. After this period, we insert $3 \times 10^{5}$ Lagrangian test particles between 50 and 150 au, with a uniform dust-to-gas mass ratio of $1 \%$ across this radial region, and we run simulations for additional 300 planetary orbits. The size of the test particles is uniformly distributed between $1 \mu \mathrm{m}$ and $1 \mathrm{~cm}$ in logarithmic space. The mass of each test particle is determined such that, at each radius in the disk, the dust mass is distributed over a range of dust sizes, from $1 \mu \mathrm{m}$ and $1 \mathrm{~cm}$ to have the mass per interval in $\log (s)$ be proportional to $s^{0.5}$, consistent with a dust size distribution of $n(s) \propto s^{-3.5}$. We assume a dust bulk density of $\rho_{s}=1.26 \mathrm{~g} \mathrm{~cm}^{-3}$, which corresponds to the density of aggregates with $30 \%$ silicate matrix and $70 \%$ water ice. The bulk density is lower than that of sub- $\mu \mathrm{m}$ grains responsible for the thermalization of the stellar radiation (see Sect. 4.1), since for the small dust grains in the upper layers of the disk we do not expect ice-coating.

Recent numerical simulations of planet-disk interaction have shown that the propagation and dissipation of planet-driven spiral waves and the number and depth of associated gaps strongly depend on the thermal properties of the disk gas, in particular, the cooling timescale of the disk gas (Miranda \& Rafikov 2020; Zhang \& Zhu 2020; Weber et al. 2019; Ziampras et al. 2020). Inspired by these studies, we run three hydrodynamical simulations: (1) a locally isothermal simulation, adopting an isothermal equation of state (hereafter isothermal model); (2) an adiabatic simulation, adopting an adiabatic equation of state with an adiabatic index $\gamma=1.4$ and $\beta=1$ (hereafter $\beta=1$ model), where $\beta$ is defined as the multiplication of the cooling time $t_{\text {cool }}$ and local orbital frequency $\Omega, \beta \equiv t_{\text {cool }} \Omega$; and (3) an adiabatic simulation with $\gamma=1.4$ and $\beta=100$ (hereafter $\beta=100$ model). In $\beta=1$ and $\beta=100$ models, the disk temperature is relaxed toward the initial temperature over the cooling timescale $t_{\text {cool }}$.

\subsection{Model results}

In Fig. 5, we present two-dimensional disk surface density and particle distributions. Gas and particle distributions in a radiusazimuthal angle plane, along with azimuthally-averaged radial profiles of gas and dust surface density, are shown in Fig. 6.

In the isothermal model, the inner and outer primary spiral arms (indicated with red arrows in Fig. 6) create shocks at \pm 13 au from the planet, which corresponds to about \pm 2 scale heights at the radial location of the planet. This is in a good agreement with the shock distance predicted by linear theory (Goodman \& Rafikov 2001). A secondary spiral arm forms in the inner disk (indicated with blue arrows in Fig. 6) and creates shocks around $35 \mathrm{au}$. As these spiral arms form they generate shocks, opening gaps in the disk (Bae et al. 2017). The gas pressure has local maxima between the gaps and beyond the outer gap at 48, 70, and $90 \mathrm{au}$, around which radii particles are aerodynamically dragged and trapped. Therefore, the planet generates three rings and two gaps in the dust disk, and the planet is embedded in the middle ring. In addition, within the co-rotation region, large particles $(\sim 1 \mathrm{~cm})$ are preferentially collected around one of the Lagrangian points, L5, which locates $60^{\circ}$ behind the planet, leading to azimuthal asymmetries in the density distribution of large grains.

In the $\beta=1$ model, the planet opens a single, broad gap around its orbit. This distinct feature of single gap opening around a sub-thermal-mass planet is known to happen when $\beta \simeq 0.1-1$ as linear damping plays a more important role in angular momentum transfer than non-linear shock dissipation (Miranda \& Rafikov 2020; Zhang \& Zhu 2020). As a result, two gas pressure peaks form in this model, one at the outer edge of the inner cavity at $52 \mathrm{au}$ and the other beyond the gap at $75 \mathrm{au}$. The planet is embedded within the gap, sandwiched by two dust rings.

In the $\beta=100$ model, the disk behaves nearly adiabatically and the perturbation driven by planet-driven spiral arms is much weaker compared with the isothermal model (Miranda \& Rafikov 2020; Zhang \& Zhu 2020). A secondary spiral arm forms in the inner disk around $30 \mathrm{au}$, but the density perturbation driven by the spiral arm is much weaker compared with that seen in the isothermal model. With a finite viscosity implemented in the simulation $\left(\alpha=10^{-4}\right)$, neither primary nor secondary spiral arms open a gap. The gas pressure peaks slightly inward of the planet's orbit, at $62 \mathrm{au}$. Large particles $(\sim 1 \mathrm{~cm})$ are collected around Lagrangian point L5, similar to the isothermal model.

Based on the three models, we generate simulated continuum images. We first create 50 logarithmically-spaced grain size bins between $1 \mu \mathrm{m}$ and $1 \mathrm{~cm}$ and compute the surface density of each grain component at each simulation grid cell. We then expand the dust disk vertically, assuming that grains are in a hydrostatic equilibrium within isotropic turbulence characterized by $\alpha=10^{-4}$ and have a Gaussian distribution. With this assumption, the scale height of dust particle having size $s, H_{d}(s)$, can be 


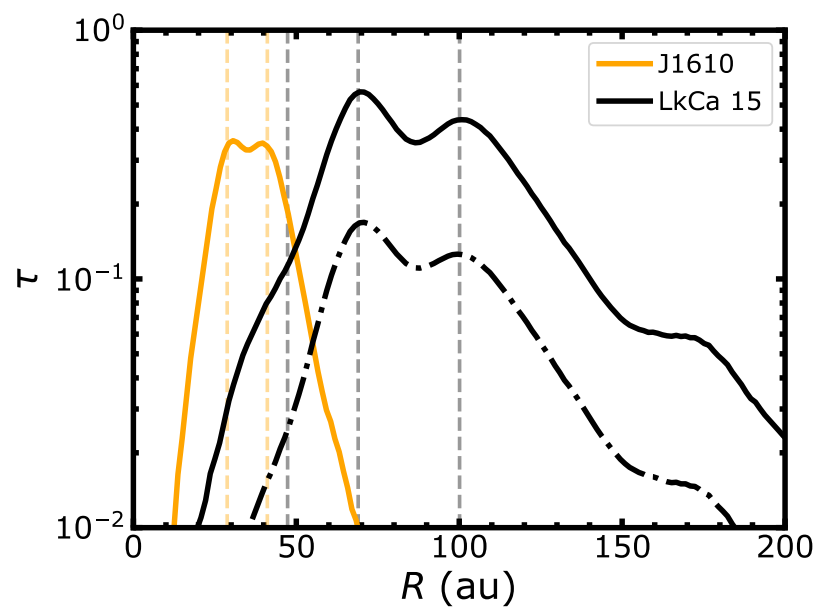

Fig. 8. Optical depth profiles of both disks derived from Eq. (5). The solid curves show the optical depth profiles assuming the temperature profile from Eq. (6). The dashed-dotted line portrays the optical depth profile of $\mathrm{LkCa} 15$ obtained with the temperature profile calculated with the radiative transfer model (see Sect. 4 and Eq. (3)). The optical depth profiles have been computed assuming absorption opacities only. The vertical dashed lines indicate the rings locations as derived from the $(u, v)$-plane modeling.

written as:

$H_{d}(s)=H \times \min \left(1, \sqrt{\frac{\alpha}{\min (\mathrm{St}, 1 / 2)\left(1+\mathrm{St}^{2}\right)}}\right)$,

where $\mathrm{St} \equiv \pi \rho_{s} s / 2 \Sigma_{\mathrm{g}}$ is the Stokes number of the particle (Birnstiel et al. 2010). The raw continuum emission is obtained with RADMC-3D (Dullemond et al. 2012), considering both absorption and anisotropic scattering using the HenyeyGreenstein approximation. We note that scattering dominates the continuum emission in the simulated observations, supporting recent explanations to low optical depths estimated in spatially resolved continuum observations (Zhu et al. 2019). We assume a distance of $158.9 \mathrm{pc}$ and disk inclination and PA of $50^{\circ}$ and $62^{\circ}$, respectively. We then convolve the raw images with the same synthesized beam used for the $\mathrm{LkCa} 15$ observation (i.e., $68 \times 47$ mas with a PA of $\left.-12.6^{\circ}\right)$ and add random noise at the same level to the observation (i.e., $6.9 \mu \mathrm{Jy}_{\text {beam }}{ }^{-1}$ ).

The resulting simulated continuum images are presented in Fig. 7. As shown, a $60 M_{\oplus}$ planet can produce diverse continuum morphology depending on the disk's cooling timescale. In the isothermal model, the planet generates three continuum rings separated by two gaps. The planet is embedded in the middle ring. In $\beta=1$ model, the planet opens a gap and two rings. The planet is embedded in the gap. In $\beta=100$ model, the disk appears as if it does not have any planets beyond the cavity; the disk is well characterized by the inner cavity and a single ring without footprints from the $60 M_{\oplus}$ planet.

\section{Discussion}

Rings in transition disks. About 40 disks that have been observed at moderate angular resolution $\left(\sim 0.2-0.4^{\prime \prime}\right)$ show evidence for dust depleted inner cavities with radius of a few tens of au (e.g., Pinilla et al. 2020). A few of these disks have been observed at about ten times better resolution and present small scale substructure beyond their cavity (e.g., Kudo et al. 2018; Pérez et al. 2019; Huang et al. 2020). All of them host multiple rings, up to four (Pérez et al. 2019), except HD 100453 that is a known binary system. In addition to rings, some targets show localized azimuthal asymmetries (Pérez et al. 2018; Dong et al. 2018). Although the number of transition disks observed at high resolution is significantly lower than those of classical disks with no cavity (e.g., Andrews et al. 2018), it seems that rings are the most common type of substructure also in transition disks as in classical disks (Huang et al. 2018). These rings are likely to be tracing the trapping of dust grains (Dullemond et al. 2018), possibly as a consequence from the presence of companions located inside the cavity. This enables to maintain a significant dust mass in the outer disk over few million years, as mm dust particles would otherwise rapidly drift towards the star (e.g., Brauer et al. 2008). While most of the rings in transition disks can be well modeled with a radial Gaussian profile, some objects clearly show a strong asymmetry in their innermost ring, with an additional shoulder located inwards (in LkCa 15 and HD 169142; this paper, Pérez et al. 2019) or outwards of the ring (as in DM Tau and GM Aur; Kudo et al. 2018; Huang et al. 2020). Higher angular resolution (and possibly longer wavelengths to avoid optical thickness) will help determining the nature of these peculiar morphologies, which may be connected to the physical mechanism carving the prominent cavities in these disks. At the same time, outer rings show shallower profiles in the outward direction. This is the case for B100 and B41 in LkCa 15 and J1610, respectively. These radial asymmetries in the intensity profile of dust rings are of particular interest to discern what is causing them. Indeed gaps generated by planets are expected to create an asymmetric pressure profile, which would lead to skewed rings in the dust (e.g., Dullemond et al. 2018). This is even more pronounced when different grain sizes of dust are considered in the modeling, with large grains well confined at the pressure maximum but smaller grains tracing the underlying gas surface density (e.g., Pinilla et al. 2018). Pinilla et al. (2018) also showed how this effect can depend on the stellar mass. A larger sample of transition disks spanning different stellar and disk properties imaged at high angular resolution would help understand the physical origin of this common feature in the intensity profiles.

Optical depth. From the intensity profiles, we compute optical depth profiles under the assumptions of a uniform opacity across the disks, and that the albedo of the dust grains is negligible. The optical depths have been computed from:

$I_{v}(R)=B_{v}(R)\left(1-\mathrm{e}^{-\tau_{v}(R)}\right)$,

with $B_{v}$ being the Planck function at the frequency of the observations, and $I_{v}$ being the azimuthally averaged intensity profile derived in Sect. 3. For LkCa 15 two midplane temperatures are used in the calculation, the one computed with the radiative transfer model discussed in Sect. 4 (see Eq. (3)), and one expression used to derive optical depth profiles in the DSHARP large program (Andrews et al. 2018; Huang et al. 2018):

$T(R)=\left(\frac{\varphi L_{*}}{8 \pi R^{2} \sigma_{\mathrm{SB}}}\right)^{0.25}$,

where $\sigma_{\mathrm{SB}}$ in the Stefan-Boltzmann constant, and $\varphi$ is the flaring angle, which we set equal to 0.02 as in Huang et al. (2018). Only this temperature profile has been used for J1610. The rings appear as marginally optically thick, with peak values ranging between 0.18 and 0.6 (depending on the exact temperature structure) for LkCa 15, and being $\sim 0.3$ for J1610. The optical depth profiles are shown in Fig. 8, where LkCa 15 is presented with two curves based on different temperature profiles. A maximum optical depth of $0.3-0.6$ is remarkably similar to the ones obtained 
in other studies (Huang et al. 2018; Facchini et al. 2019). This may be caused by the presence of very large grains with significantly high albedo, with the optical depth peak derived from Eq. (5) being a lower limit in the absence of scattering (e.g., Liu 2019; Zhu et al. 2019). Indeed, scattering is what dominates the simulated continuum images presented in Fig. 7. Observations at multiple wavelengths (more than two) are needed to estimate how important scattering can be at $\mathrm{mm}$ wavelengths (e.g., Carrasco-González et al. 2019; Huang et al. 2020) and whether it can explain the observational result of roughly uniform maximum optical depth in rings and inner regions across different disks. Stammler et al. (2019) instead proposed that a maximum optical depth of $\sim 0.5$ can be driven by streaming instability self-regulating the dust-to-gas ratio within pressure maxima (see also Dullemond et al. 2018), which would be indirect evidence of planetesimal formation with the dust rings observed with ALMA. To disentangle between these different possibilities, multi-wavelengths observations at high spatial resolution are needed.

Dust masses and planetesimal formation. From the optical depth profiles, it is possible to compute the amount of dust present in the rings by knowing that $\Sigma_{\mathrm{d}}(R)=\tau_{v} / \kappa$. For a given temperature profile and a given opacity, these values represent a lower limit, since we assume negligible albedo of the dust grains. The largest uncertainties lay in the dust opacity estimates, with values being unknown within $1 \mathrm{dex}$ from the value we assume. From the opacity laws used in Sect. 4, we have an absorption opacity of $2.6 \mathrm{~cm}^{2} \mathrm{~g}^{-1}$ at $1.3 \mathrm{~mm}$. For LkCa 15 we compute the mass of B47 and B69 together, integrating the optical depth profile between 19 and 88 au. As for B100, we compute the dust mass between 88 and $150 \mathrm{au}$. By using the temperature profile in Eq. (3), we obtain dust masses of 51 and $182 M_{\oplus}$ for B47+B69 and $\mathrm{B} 100$, respectively, whereas using the temperature profile of Eq. (6) leads to dust masses of 32 and $113 M_{\oplus}$ for the same regions. We integrate the optical depth profile of J1610 between 9.7 and 35.8 au for B29 and between 35.8 and 72.2 au for B41, obtaining 10 and $19 M_{\oplus}$, respectively.

These disks are still continuing to retain a large amount of dust mass in this phase of their evolution, and planetesimal formation within these radially confined regions may still be operational via streaming instability (e.g., Youdin \& Goodman 2005; Johansen et al. 2007). In order to assess whether planetesimal formation via streaming instability may be occurring within the dust rings, we need to compare the gas with the dust surface density within the dust rings. We use the dust surface density computed from the optical depth profile, and we compare it with the upper limit of the gas surface density at the location of the dust rings. In order to have a physically motivated upper limit, we require that the disk is not locally gravitationally unstable, since this would develop spiral structures that we do not see in the ALMA images (e.g., Kratter \& Lodato 2016):

$Q_{\text {Toomre }} \equiv \frac{c_{\mathrm{s}} \Omega_{\mathrm{K}}}{\pi G \Sigma_{\mathrm{g}}}>2$,

where $c_{\mathrm{S}}$ is the local sound-speed, $\Omega_{\mathrm{K}}$ is the Keplerian angular velocity and $\Sigma_{\mathrm{g}}$ is the gas surface density. The sound speed is estimated from Eq. (6). With these calculations, we find that for $\mathrm{LkCa} 15$, the dust rings B69 and B100 show that $\Sigma_{\mathrm{g}} / \Sigma_{\mathrm{d}}<[68,62]$ at their intensity peaks, respectively. As for J1610, B29 and B41 present $\Sigma_{\mathrm{g}} / \Sigma_{\mathrm{d}}<[190,138]$. If local turbulence is driven by the streaming instability itself, planetesimals are expected to form whenever $\Sigma_{\mathrm{g}} / \Sigma_{\text {dust }} \lesssim 50$ (e.g., Bai \& Stone 2010), in which case the upper limits we obtained for LkCa 15 are intriguingly close to this value. If instead an additional turbulence component is present, the vertical settling of dust grains is reduced and the nominal condition for planetesimal formation to occur is less stringent (e.g., Gole et al. 2020). We note, however, that the co-existence of vertical turbulent motions as driven by other instabilities as the vertical shear instability or magneto-rotational instability may promote rather than suppress planetesimal formation (Johansen et al. 2007; Schäfer et al. 2020).

One possible way to derive information about the gas turbulence in disks is to determine how radially (or vertically) confined dust grains are in disks. In the following, we look at the radial concentration of dust particles at the rings locations in the assumption that these are dust traps. We follow the arguments of Dullemond et al. (2018) to characterize radially resolved rings in the DSHARP sample. First of all, we compute how radially confined the dust rings are, in order to have a lower limit on the combination of the turbulence viscosity $\alpha$ and the characteristic grain size within the rings. Dullemond et al. (2018) showed that:

$$
\frac{\alpha}{\mathrm{St}}=\left[\left(\frac{\sigma}{\sigma_{\mathrm{d}}}\right)^{2}-1\right]^{-1} \text {, }
$$

where St is the Stokes number of the characteristic dust grain size entrained in the ring (we can think at the characteristic size as the one dominating the emission at the observed wavelength), and $\sigma$ and $\sigma_{\mathrm{d}}$ are the widths of the gas and dust components of the rings, approximated as radial Gaussian profiles in the proximity of the rings. While Dullemond et al. (2018) computed the dust Gaussian widths by fitting the rings from the imaged radial intensity profiles, we use the ones obtained from the $(u, v)$-plane fitting (values are reported in Table 2). As for the gas Gaussian widths, we determine lower and upper limits using the same arguments as in Dullemond et al. (2018). The lower limit $\sigma_{\text {min }}$ is the maximum value between the local pressure scale height $H$ and the Gaussian dust width. To estimate the pressure scaleheight, we use the temperature profile from Eq. (6), and derive $H$ knowing that:

$H(R)=\sqrt{\frac{k_{\mathrm{B}} T}{\mu m_{\mathrm{H}}} \frac{R^{3}}{G M_{*}},}$

with $\mu$ being the molecular weight and $m_{\mathrm{H}}$ being the hydrogen mass. The upper limit $\sigma_{\max }$ is set such that the distance between the two rings is larger than the full-width-half-maximum of the gas Gaussian profile. Given that the rings in J1610 are likely unresolved even in the $(u, v)$-plane analysis (see Sect. 3.2), we can determine upper limits only on $\alpha /$ St. In particular, we obtain an upper limit of $\alpha / \mathrm{St}_{\max } \gtrsim 15.3$ for B41 in J1610, and a lower limit of $\alpha / \mathrm{St}_{\min } \lesssim 0.29$ for $\mathrm{B} 69$ in $\mathrm{LkCa} 15$. These values are in general agreement with the results on the DSHARP sample (Dullemond et al. 2018), possibly suggesting that dust confinement within rings in full or transition disks may be similar. Additional information could be derived by having higher angular resolution observations on $\mathrm{J} 1610$ to radially resolve the rings, having multi-wavelengths datasets to translate St into a physical grain size, and having direct measurements of the ring widths in the gas component determining the pressure profile through kinematical analysis (e.g., Teague et al. 2018; Rosotti et al. 2020).

Planet-disk interactions. In Sect. 4 we explored the possibility that multiple rings in transition disks are footprints of an embedded planet, while we have also shown that disk thermodynamics alone can drastically change the morphology in 
the mm-continuum emission. We consider what the cooling timescale might be in disks. Adopting the gray atmosphere approximation (Hubeny 1990) and assuming that the disk temperature is dominated by stellar irradiation, the cooling timescale can be estimated as (see e.g., Zhu et al. 2015):

$t_{\mathrm{cool}}=\frac{3 \Sigma_{\mathrm{g}} c_{V}}{64 \sigma_{\mathrm{SB}} T_{\mathrm{mid}}^{3}} \frac{1+\tau_{R}^{2}}{\tau_{R}}$

or:

$$
\begin{aligned}
\beta= & t_{\text {cool }} \Omega \\
= & 0.024\left(\frac{\Sigma_{\mathrm{g}}}{35 \mathrm{~g} \mathrm{~cm}^{-2}}\right)\left(\frac{T_{\mathrm{mid}}}{34 \mathrm{~K}}\right)^{-3}\left(\frac{M_{*}}{1.25 M_{\odot}}\right)^{0.5}\left(\frac{R}{70 \mathrm{au}}\right)^{-1.5} \\
& \times\left(\frac{1+\tau_{R}^{2}}{\tau_{R}}\right),
\end{aligned}
$$

where $c_{V} \equiv(\gamma-1) \mathcal{R} / \mu$ is the specific heat capacity of gas, $\mathcal{R}$ is the gas constant, $\mu=2.4$ is the mean molecular weight, and $\tau_{R}=\kappa_{R} \Sigma_{\mathrm{g}} / 2$. With the initial disk conditions described in Sect. 4 and a range of Rosseland mean opacity value of $\kappa_{R}=0.1-10 \mathrm{~cm}^{2} \mathrm{~g}^{-1}$, the cooling parameter $\beta$ at 70 au in the LkCa 15 disk is $\sim 0.07-4.3$. We adopted a range of Rosseland mean opacity value as it requires a proper knowledge of the dust size distribution which depends upon a variety of physical processes and parameters, including the dust fragmentation velocity, the level of disk turbulence, dust internal density, and underlying gas density and temperature (Birnstiel et al. 2018). In addition, the dust-to-gas mass ratio as well as the dust size distribution can evolve over time as a planet redistributes dust particles around its orbit. Nevertheless, this simple estimation shows that deviations from the isothermality, which previous studies have shown to occur for $\beta \gtrsim 0.01$ (Miranda \& Rafikov 2020; Zhang $\&$ Zhu 2020), are likely to happen in protoplanetary disks and should be considered when modeling their observations with hydrodynamical simulations.

In addition to disk's thermodynamic properties, many other parameters and physical processes also play a role in determining the location and appearance of the mm-rings generated by the planet-disk interactions. Among others, this includes the underlying gas density structure, disk temperature, turbulence, planet mass, grain properties, dust feedback, and orbital migration. This shows how challenging it is to retrieve planet mass and location by analysing the properties of rings in disks (in the hypothesis that these are the outcome of planet-disk interactions). At the same time, the resemblance of the morphological features observed in disks with the results of hydrodynamical simulations of planets-disk interactions is surprising, and suggests that rings in disks are (at least in part) generated by planets. The level and morphology of substructures in transition disks, and in particular LkCa 15 and J1610, suggest that the observed morphology can be caused by low mass planets that are formed within massive dust rings. Even though we have been agnostic on the mechanism generating the inner cavity by using a prescribed gas surface density, in the assumption of massive planets clearing the inner cavity it is reasonable to speculate that the resulting dust trapping can generate secondary planet formation from the inside-out, with planetary cores forming in dust traps created by the inner and more massive planet. However, we point out that planetary core formation via pebble accretion within dust rings at large distances from the central star ( $\gg 10 \mathrm{au}$ ) may be challenging due to the long dynamical timescales (e.g., Morbidelli 2020).
Snow lines. While our model shows that a low mass planet is able to reproduce the observed features, planet-disk interactions are not the only means to form annular structure in disks. Among the numerous models proposed, dust properties and opacities are expected to vary at the ice lines of abundant chemical species, with physical mechanisms such as outward diffusion and sintering accentuating the contrast outside the icelines (Stevenson \& Lunine 1988; Okuzumi et al. 2016). While surveys of disk annular substructures did not find any correlation between the location of the rings with estimated ice lines location in disks when using simple temperature prescriptions (Huang et al. 2018; Long et al. 2018), indicating a different origin for most rings, some of them might still be induced at condensation fronts. $\mathrm{N}_{2} \mathrm{H}^{+}$is a molecule known to be abundant between the CO and $\mathrm{N}_{2}$ ice lines (e.g., Qi et al. 2013; van 't Hoff et al. 2017), and most importantly it traces the location of the ice lines without needing to assume a temperature or a desorption temperature of the two molecules. Qi et al. (2019) observed $\mathrm{N}_{2} \mathrm{H}^{+}$in $\mathrm{LkCa} 15$, showing that this ion is particularly abundant between $58_{-10}^{+6}$ and $88_{-4}^{+6}$ au. These radii are just inwards of the B69 and $\mathrm{B} 100$ rings, respectively, and it may suggest that the two rings are formed by condensation of these two abundant molecules. However, as noted by Huang et al. (2020) in the similar case of GM Aur, the scattered light data by Thalmann et al. (2016) do not show clear rings co-located with the ones observed in ALMA. This seems to favour a planetary origin of the rings, rather than chemical, since in the first case rings in scattered light are predicted to be less pronounced than at $\mathrm{mm}$ wavelengths, whereas in the second case the opposite is expected (Pinilla et al. 2017). Interestingly, both GM Aur and LkCa 15 show a faint outer ring in $\mathrm{N}_{2} \mathrm{H}^{+}$emission at 200 and 220 au, respectively (Qi et al. 2019), and at the same time show an outer shallow ring in the continuum intensity profile interior of the molecular ring (at $\sim 170$ and $\sim 175 \mathrm{au}$, respectively), as shown in this paper and in Huang et al. (2020). It is likely that the outer $\mathrm{N}_{2} \mathrm{H}^{+}$ring is related to a radial thermal inversion in the proximity of the dust outer radius which in turns leads to $\mathrm{CO}$ being released into gas phase again (e.g., Facchini et al. 2017); however, it would be worth exploring whether there is any potential connection between outer $\mathrm{CO}$ snow-lines and shallow rings close to edge of the mm-emission, analogously to what is expected to happen at the inner COsnowline but in the opposite radial direction. No observations of molecular species rather than $\mathrm{CO}$ are available for J1610, thus estimates of ice lines locations would still suffer from the large uncertainties attributed to disk temperature retrieval.

It is worth mentioning that there can be alternative scenarios to the planet-disk interaction and snow lines cases. For example, Pinilla et al. (2016) modeled the grain size evolution in pressure maxima generated by a dead-zone (i.e. a radial discontinuity in the viscosity parameter governing radial advection of gas parcels in a disk Gammie 1996; Regály et al. 2012; Flock et al. 2015), showing that substructure in the dust properties at the dead zone edge may occur on Myr timescales (see their Fig. 4). The present observations are not capable of distinguishing between these different models.

Inner disk and azimuthal asymmetries. Both LkCa 15 and J610 exhibit an inner disk via an IR excess (e.g., Espaillat et al. 2008; Ansdell et al. 2016), and LkCa 15 shows detectable mm continuum emission at the center of the cavity in the ALMA image. Based on the assumption that this is due to dust in the proximity of the star, by using the same dust opacity as above and a temperature of $100 \mathrm{~K}$, the inner disk shows a dust mass of $\sim 0.4$ Moon masses in the optically thin approximation. Since 
the emission seems unresolved at our spatial resolution, the flux density of the inner disk can be related to an inner disk size in the optically thick approximation, with a radius of $\sim 0.15$ au for a temperature of $100 \mathrm{~K}$. By using a gas-to-dust ratio of 100 , the estimated mass in gas of the inner disk emitting at $1.3 \mathrm{~mm}$ is $\sim 1.5 \times 10^{-6} M_{\odot}$, which would be entirely accreted in $\sim 3000 \mathrm{yr}$ at the current accretion rate, in a too short timescale to be realistic. This can be easily overcome since the mass estimate we computed is a lower limit in the optically thin assumption, and also because the inner disk is likely constantly replenished by the outer disk through material flowing into the cavity.

Interestingly, both Lkca 15 and J1610 are known to host variability in their optical light curve, and have been both classified as dippers (e.g., Ansdell et al. 2016; Alencar et al. 2018). Dippers are pre-main sequence stars that host quasi-periodic or episodic dimming in the lightcurve, with durations of about one day (e.g., Alencar et al. 2010; Cody et al. 2014). Different models have been invoked to explain the photometric behaviour, in general requiring small amount of dust close to the dust sublimation radius to be interposed along the line of sight generating partial occultation of the central star. Among many, proposed models are magnetospheric accretion lifting dust along the accretion column (Bouvier et al. 1999), dust laden winds, thermal instabilities or misaligned inner disks. Many of these mechanisms rely on the inner disk to be close to edge-on (e.g., Bodman et al. 2017; Cody \& Hillenbrand 2018), in order for the mechanism to be able to displace dust along the line of sight. However, Ansdell et al. (2020) have shown that the distribution of outer disk $(R \gtrsim 10 \mathrm{au})$ inclinations of dippers as retrieved by ALMA imaging is isotropic, suggesting that outer disk inclination is not a relevant parameter to determine the "dipper" status of young stars. In both disks analysed in this paper, the residuals shown in Fig. 4 show asymmetric features. Such features could be due both to thermal effects (and thus shadowing) or to local surface density effects, for example generated by decaying vortexes in low turbulence environments. The $\mathrm{LkCa} 15$ disk shows a north-west region that is brighter than the south-east, in agreement with the scattered light image and model by Thalmann et al. (2015). At the same time, J1610 presents evident negative residuals at PA of $\sim 55^{\circ}$ and $\sim 190^{\circ}$, and strong positive residuals in the southeast side of the disk. This feature is strikingly similar to what is observed in scattered light images of disks interpreted as hosting a misaligned inner disk (e.g., Marino et al. 2015; Benisty et al. 2018; Muro-Arena et al. 2020) with a misalignment of $\lesssim 30^{\circ}$ (Facchini et al. 2018). Dedicated radiative transfer models and analysis of the gas kinematics in the inner regions of the central cavity will be key in confirming and characterizing the presence of misaligned inner disks in both systems.

\section{Conclusions}

In this paper, we present and analyze high angular resolution ALMA continuum observations of two transition disks, namely, LkCa 15 and 2MASS J16100501-2132318. Our main results are summarized as follows:

- Both disks are structured and the broad rings previously imaged at lower resolution resolve in multiple rings. LkCa 15 has rings located at $\sim 47,69$ and $100 \mathrm{au}$, with widths $\sim 9,6$, $14 \mathrm{au}$, respectively, and possibly another faint ring located further out at $\sim 175 \mathrm{au}$. J1610 shows two rings at $\sim 29$ and $41 \mathrm{au}$, with widths of $\sim 2,3$ au at the current resolution. Only the two outer rings in $\mathrm{LkCa} 15$ are radially resolved at the present angular resolution. Both disks also host an extended component covering a broad range of radii.
- Rings appear to be marginally optically thick assuming negligible albedo of the dust grains, with optical depths at peaks ranging between $\sim 0.3$ and 0.6. All rings are more massive than $10 M_{\oplus}$, and can be as massive as $\gtrsim 150 M_{\oplus}$. The properties of the rings are similar to the ones observed in protoplanetary disks with no cavity (i.e., not transition) when imaged at high angular resolution.

- While evidence for the presence of an inner disk has already been inferred from the near-IR excess in the SED (e.g., Espaillat et al. 2008), only LkCa 15 shows evidence of a compact millimeter emission inside the cavity indicating a dust-laden inner disk. At our sensitivity, we do not detect such inner emission in J1610.

- Both disks show azimuthal asymmetries in the brightness temperature distribution, and they resemble theoretical predictions of shadowing from misaligned inner disks. Increasing the sample of transition disks hosting dipper stars may help elucidate the link between inner and outer disks in objects with dynamically variable inner regions.

- Comparison with hydrodynamical simulations show that a planet embedded in the outer disk can lead to the formation of multiple rings beyond a cavity. However, we find that the thermodynamics can dramatically affect the number and appearance of these rings, suggesting that planet and disk properties inferred from models should be interpreted with caution. The uncertainty of the disk cooling adds to other uncertainties from planet migration, planet formation timescales, disk turbulence, among others that have been previously studied.

- The radii of the two prominent rings in $\mathrm{LkCa} 15$ are in broad agreement with observations locating the $\mathrm{CO}$ and $\mathrm{N}_{2}$ snowlines from $\mathrm{N}_{2} \mathrm{H}^{+}$observations (Qi et al. 2019), suggesting that chemistry may be causing (or facilitating) the intensity contrast observed in the radial profile.

- The rings in both disks appear as favourable locations where streaming instability can occur, leading to planetesimal (and possibly subsequent planetary core) formation. The qualitative agreement between the hydrodynamical simulations and the observed morphology suggests that massive rings in transition disks may be the best environments to characterize the conditions of formation of planetary cores. If the inner cavity is cleared by a yet unseen massive planet, we may be witnessing a second-generation planet formation event triggered by the dust trap generated by the inner body.

Future observations at even higher angular resolution may pin down the nature of the inner shoulder (B49) observed in LkCa 15. At the same time, high angular resolution observations can provide better constraints on the efficiency of dust trapping and on the dust diffusion within rings. Multi-frequency high resolution observations and gas kinematics studies will be particularly beneficial in shedding light on the dust trapping mechanisms in transition disks.

Acknowledgements. We are thankful to Marco Tazzari, Leonardo Testi and Antonella Natta for fruitful discussions, and to the anonymous referee whose comments helped improving the manuscript. This paper makes use of the following ALMA data: ADS/JAO.ALMA\#2018.1.01255.S. ALMA is a partnership of ESO (representing its member states), NSF (USA), and NINS (Japan), together with NRC (Canada), NSC and ASIAA (Taiwan), and KASI (Republic of Korea), in cooperation with the Republic of Chile. The Joint ALMA Observatory is operated by ESO, AUI/NRAO, and NAOJ. S.F. acknowledges an ESO Fellowship, and is grateful to JAO for hosting a month long visit which was greatly beneficial for completing the paper. M.B. acknowledges funding from ANR of France under contract number ANR-16-CE31-0013 (Planet Forming Disks). J.B. acknowledges support by NASA through the NASA Hubble Fellowship grant \#HST-HF2-51427.001-A awarded by the Space Telescope Science Institute, 
which is operated by the Association of Universities for Research in Astronomy, Incorporated, under NASA contract NAS5-26555, and computing resources provided by the NASA High-End Computing Program through the NASA Advanced Supercomputing Division at Ames Research Center. P.P. acknowledges support provided by the Alexander von Humboldt Foundation in the framework of the Sofja Kovalevskaja Award endowed by the Federal Ministry of Education and Research. A.W.M. was partially supported through NASA's Astrophysics Data Analysis Program (80NSSC19K0583). This project has received funding from the European Union's Horizon 2020 research and innovation programme under the Marie Sklodowska-Curie grant agreement No 823823 (Dustbusters RISE project), and was partly supported by the Deutsche Forschungs-gemeinschaft (DFG, German Research Foundation) - Ref no. FOR 2634/1 TE 1024/1-1.

\section{References}

Alencar, S. H. P., Teixeira, P. S., Guimarães, M. M., et al. 2010, A\&A, 519, A88

Alencar, S. H. P., Bouvier, J., Donati, J. F., et al. 2018, A\&A, 620, A195

Alexander, R. D., Clarke, C. J., \& Pringle, J. E. 2006, MNRAS, 369, 229

Allard, F., Homeier, D., Freytag, B., et al. 2013, Mem. Soc. Astron. It. Suppl., 24,128

Andrews, S. M. 2020, ARA\&A, submitted [arXiv:2001.05007]

Andrews, S. M., Rosenfeld, K. A., Wilner, D. J., \& Bremer, M. 2011, ApJ, 742 L5

Andrews, S. M., Huang, J., Pérez, L. M., et al. 2018, ApJ, 869, L41

Ansdell, M., Gaidos, E., Rappaport, S. A., et al. 2016, ApJ, 816, 69

Ansdell, M., Gaidos, E., Hedges, C., et al. 2020, MNRAS, 492, 572

Bae, J., Zhu, Z., \& Hartmann, L. 2017, ApJ, 850, 201

Bae, J., Zhu, Z., Baruteau, C., et al. 2019, ApJ, 884, L41

Bai, X.-N., \& Stone, J. M. 2010, ApJ, 722, L220

Barenfeld, S. A., Carpenter, J. M., Ricci, L., \& Isella, A. 2016, ApJ, 827, 142

Baruteau, C., \& Masset, F. 2008a, ApJ, 672, 1054

Baruteau, C., \& Masset, F. 2008b, ApJ, 678, 483

Baruteau, C., \& Zhu, Z. 2016, MNRAS, 458, 3927

Baruteau, C., Barraza, M., Pérez, S., et al. 2019, MNRAS, 486, 304

Benisty, M., Juhász, A., Facchini, S., et al. 2018, A\&A, 619, A171

Birnstiel, T., Dullemond, C. P., \& Brauer, F. 2010, A\&A, 513, A79

Birnstiel, T., Dullemond, C. P., Zhu, Z., et al. 2018, ApJ, 869, L45

Bitsch, B., Lambrechts, M., \& Johansen, A. 2015, A\&A, 582, A112

Bodman, E. H. L., Quillen, A. C., Ansdell, M., et al. 2017, MNRAS, 470, 202

Bouvier, J., Chelli, A., Allain, S., et al. 1999, A\&A, 349, 619

Brauer, F., Dullemond, C. P., \& Henning, T. 2008, A\&A, 480, 859

Brown, J. M., Blake, G. A., Qi, C., et al. 2009, ApJ, 704, 496

Carrasco-González, C., Sierra, A., Flock, M., et al. 2019, ApJ, 883, 71

Casassus, S., \& Pérez, S. 2019, ApJ, 883, L41

Casassus, S., Avenhaus, H., Pérez, S., et al. 2018, MNRAS, 477, 5104

Cazzoletti, P., van Dishoeck, E. F., Pinilla, P., et al. 2018, A\&A, 619, A161

Cody, A. M., \& Hillenbrand, L. A. 2018, AJ, 156, 71

Cody, A. M., Stauffer, J., Baglin, A., et al. 2014, AJ, 147, 82

Currie, T., Marois, C., Cieza, L., et al. 2019, ApJ, 877, L3

de Val-Borro, M., Edgar, R. G., Artymowicz, P., et al. 2006, MNRAS, 370,529

Dodson-Robinson, S. E., \& Salyk, C. 2011, ApJ, 738, 131

Donati, J. F., Bouvier, J., Alencar, S. H., et al. 2019, MNRAS, 483, L1

Dong, R., Liu, S.-y., Eisner, J., et al. 2018, ApJ, 860, 124

Draine, B. T., \& Lee, H. M. 1984, ApJ, 285, 89

Dullemond, C. P., Juhasz, A., Pohl, A., et al. 2012, RADMC-3D: A multi-purpose radiative transfer tool

Dullemond, C. P., Birnstiel, T., Huang, J., et al. 2018, ApJ, 869, L46

Espaillat, C., Calvet, N., Luhman, K. L., Muzerolle, J., \& D’Alessio, P. 2008, ApJ, 682, L125

Evans, D. W., Riello, M., De Angeli, F., et al. 2018, A\&A, 616, A4

Facchini, S., Birnstiel, T., Bruderer, S., \& van Dishoeck, E. F. 2017, A\&A, 605, A16

Facchini, S., Juhász, A., \& Lodato, G. 2018, MNRAS, 473, 4459

Facchini, S., van Dishoeck, E. F., Manara, C. F., et al. 2019, A\&A, 626, L2

Flock, M., Ruge, J. P., Dzyurkevich, N., et al. 2015, A\&A, 574, A68

Foreman-Mackey, D., Hogg, D. W., Lang, D., \& Goodman, J. 2013, PASP, 125, 306

Gaia Collaboration (Brown, A. G. A., et al.) 2018, A\&A, 616, A1

Gammie, C. F. 1996, ApJ, 457, 355

Garufi, A., Benisty, M., Pinilla, P., et al. 2018, A\&A, 620, A94

Gole, D. A., Simon, J. B., Li, R., Youdin, A. N., \& Armitage, P. J. 2020, ApJ, submitted [arXiv:2001.10000]

Goodman, J., \& Rafikov, R. R. 2001, ApJ, 552, 793

Gorti, U., \& Hollenbach, D. 2009, ApJ, 690, 1539

Henden, A. A., Levine, S. E., Terrell, D., Smith, T. C., \& Welch, D. 2012, JAAVSO, 40, 430
Huang, J., Andrews, S. M., Dullemond, C. P., et al. 2018, ApJ, 869, L42 Huang, J., Andrews, S. M., Dullemond, C. P., et al. 2020, ApJ, 891, 48 Hubeny, I. 1990, ApJ, 351, 632

Isella, A., Pérez, L. M., \& Carpenter, J. M. 2012, ApJ, 747, 136

Isella, A., Chandler, C. J., Carpenter, J. M., Pérez, L. M., \& Ricci, L. 2014, ApJ, 788,129

Isella, A., Huang, J., Andrews, S. M., et al. 2018, ApJ, 869, L49

Isella, A., Benisty, M., Teague, R., et al. 2019, ApJ, 879, L25

Jin, S., Isella, A., Huang, P., et al. 2019, ApJ, 881, 108

Johansen, A., Oishi, J. S., Mac Low, M.-M., et al. 2007, Nature, 448, 1022

Jorsater, S., \& van Moorsel, G. A. 1995, AJ, 110, 2037

Kratter, K., \& Lodato, G. 2016, ARA\&A, 54, 271

Kraus, A. L., \& Ireland, M. J. 2012, ApJ, 745, 5

Kudo, T., Hashimoto, J., Muto, T., et al. 2018, ApJ, 868, L5

Li, A., \& Greenberg, J. M. 1997, A\&A, 323, 566

Liu, H. B. 2019, ApJ, 877, L22

Long, F., Pinilla, P., Herczeg, G. J., et al. 2018, ApJ, 869, 17

Mann, A. W., Newton, E. R., Rizzuto, A. C., et al. 2016, AJ, 152, 61

Marino, S., Perez, S., \& Casassus, S. 2015, ApJ, 798, L44

Masset, F. 2000, A\&AS, 141, 165

McMullin, J. P., Waters, B., Schiebel, D., Young, W., \& Golap, K. 2007, ASP Conf. Ser., 376, 127

Mendigutía, I., Oudmaijer, R. D., Schneider, P. C., et al. 2018, A\&A, 618, L9

Miranda, R., \& Rafikov, R. R. 2020, ApJ, 892, 65

Morbidelli, A. 2020, A\&A, 638, A1

Muiños, J. L., \& Evans, D. W. 2014, Astron. Nachr., 335, 367

Muro-Arena, G. A., Benisty, M., Ginski, C., et al. 2020, A\&A, 635, A121

Oh, D., Hashimoto, J., Tamura, M., et al. 2016, PASJ, 68, L3

Okuzumi, S., Momose, M., Sirono, S.-i., Kobayashi, H., \& Tanaka, H. 2016, ApJ, 821,82

Ormel, C. W., \& Klahr, H. H. 2010, A\&A, 520, A43

Owen, J. E., Ercolano, B., Clarke, C. J., \& Alexander, R. D. 2010, MNRAS, 401, 1415

Panagia, N., \& Felli, M. 1975, A\&A, 39, 1

Pérez, L. M., Benisty, M., Andrews, S. M., et al. 2018, ApJ, 869, L50

Pérez, S., Casassus, S., Baruteau, C., et al. 2019, AJ, 158, 15

Piétu, V., Dutrey, A., Guilloteau, S., Chapillon, E., \& Pety, J. 2006, A\&A, 460, L43

Pinilla, P., Benisty, M., \& Birnstiel, T. 2012, A\&A, 545, A81

Pinilla, P., Flock, M., Ovelar, M. d. J., \& Birnstiel, T. 2016, A\&A, 596, A81

Pinilla, P., Pohl, A., Stammler, S. M., \& Birnstiel, T. 2017, ApJ, 845, 68

Pinilla, P., Tazzari, M., Pascucci, I., et al. 2018, ApJ, 859, 32

Pinilla, P., Pascucci, I., \& Marino, S. 2020, A\&A, 635, A105

Pinte, C., Price, D. J., Ménard, F., et al. 2020, ApJ, 890, L9

Qi, C., Öberg, K. I., Wilner, D. J., et al. 2013, Science, 341, 630

Qi, C., Öberg, K. I., Espaillat, C. C., et al. 2019, ApJ, 882, 160

Regály, Z., Juhász, A., Sándor, Z., \& Dullemond, C. P. 2012, MNRAS, 419, 1701

Reynolds, S. P. 1986, ApJ, 304, 713

Rice, W. K. M., Armitage, P. J., Wood, K., \& Lodato, G. 2006, MNRAS, 373, 1619

Rizzuto, A. C., Ireland, M. J., \& Kraus, A. L. 2015, MNRAS, 448, 2737

Rodriguez, J. E., Ansdell, M., Oelkers, R. J., et al. 2017, ApJ, 848, 97

Rosotti, G. P., Benisty, M., Juhász, A., et al. 2019, MNRAS, 2689, 0

Rosotti, G. P., Teague, R., Dullemond, C., Booth, R. A., \& Clarke, C. 2020, MNRAS, 495, 173

Sallum, S., Follette, K. B., Eisner, J. A., et al. 2015, Nature, 527, 342

Schäfer, U., Johansen, A., \& Banerjee, R. 2020, A\&A, 635, A190

Siess, L., Dufour, E., \& Forestini, M. 2000, A\&A, 358, 593

Skrutskie, M. F., Cutri, R. M., Stiening, R., et al. 2006, AJ, 131, 1163

Stammler, S. M., Drążkowska, J., Birnstiel, T., et al. 2019, ApJ, 884, L5

Stevenson, D. J., \& Lunine, J. I. 1988, Icarus, 75, 146

Tazzari, M. 2017, Mtazzari/Uvplot: V0.1.1

Tazzari, M., Beaujean, F., \& Testi, L. 2018, MNRAS, 476, 4527

Teague, R., Bae, J., Bergin, E. A., Birnstiel, T., \& Foreman-Mackey, D. 2018, ApJ, 860, L12

Thalmann, C., Mulders, G. D., Janson, M., et al. 2015, ApJ, 808, L41

Thalmann, C., Janson, M., Garufi, A., et al. 2016, ApJ, 828, L17

van der Marel, N., van Dishoeck, E. F., Bruderer, S., Pérez, L., \& Isella, A. 2015, A\&A, 579, A106

van 't Hoff, M. L. R., Walsh, C., Kama, M., Facchini, S., \& van Dishoeck, E. F. 2017, A\&A, 599, A101

Walter, F., Brinks, E., de Blok, W. J. G., et al. 2008, AJ, 136, 2563

Weber, P., Pérez, S., Benítez-Llambay, P., et al. 2019, ApJ, 884, 178

Youdin, A. N., \& Goodman, J. 2005, ApJ, 620, 459

Zhang, S., \& Zhu, Z. 2020, MNRAS, 493, 2287

Zhu, Z., Dong, R., Stone, J. M., \& Rafikov, R. R. 2015, ApJ, 813, 88

Zhu, Z., Zhang, S., Jiang, Y.-F., et al. 2019, ApJ, 877, L18

Ziampras, A., Kley, W., \& Dullemond, C. P. 2020, A\&A, 637, A50 


\section{Appendix A: Stellar properties of $\mathrm{J} 1610$}

To determine the luminosity of J1610, we fit the spectral-energy distribution following the methodology from Mann et al. (2016), which we briefly summarize here. We use the spectra taken in Ansdell et al. (2016), which we absolutely calibrate using literature photometry from 2MASS (Skrutskie et al. 2006), Gaia DR2 (Evans et al. 2018), APASS (Henden et al. 2012), and CMC15 (Muiños \& Evans 2014). We simultaneously fit for reddening by comparing the spectrum to un-reddened templates of stars in nearby young moving groups observed with the same instruments and setup. We fill in gaps in the observed spectra (e.g., beyond $2.2 \mu \mathrm{m}$ ) by interpolating over a grid of BTSETTL model atmospheres (Allard et al. 2013). We calculate the bolometric flux from the integrated and un-reddened spectrum, and the luminosity from the bolometric flux and Gaia DR2 distance. We show the best-fit reddened spectrum in Fig. A.1. Final uncertainties account for errors in the flux calibration of the spectra, variability in the observed photometry, and differences in reddening from different selections of the un-reddened template. Our final value is $L_{*}=0.46 \pm 0.03 L_{\odot}$, with $E(B-V)=$ $0.26 \pm 0.06$, and $T_{\text {eff }}=3950 \pm 80 \mathrm{~K}$.

\section{Appendix B: Radial profiles and visibility modeling}

Figure B.1 shows the azimuthally averaged intensity profiles of $\mathrm{LkCa} 15$ and $\mathrm{J} 1610$ in $\log$ scale to highlight the profile at large radii. Figure B.2 shows the re-centered and deprojected visibilities of the data with the best fit model.

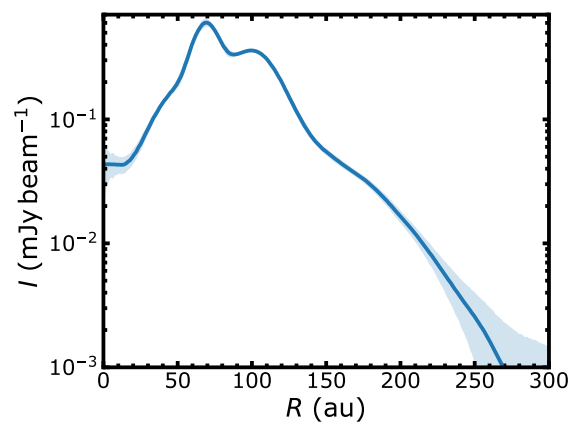

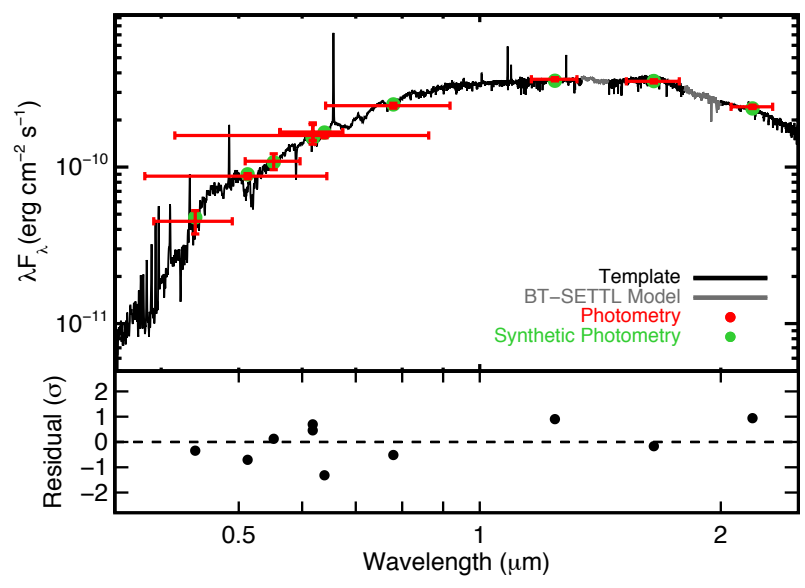

Fig. A.1. Best-fit spectral-energy distribution for J1610 created by generating synthetic photometry (green) from our observed spectra (black) and model atmosphere (grey) and locking it to the literature photometry (red). Vertical error bars on the observed photometry correspond to uncertainties (include an estimate of the stellar variability) and horizontal errors represent the width of the filter profile. Bottom panel: residual photometry in units of standard deviations. We de-redden the calibrated spectrum before computing the bolometric flux, but the spectrum and photometry shown here are still reddened.

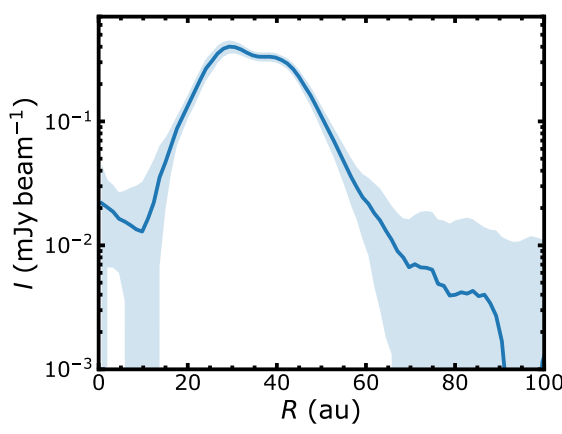

Fig. B.1. Deprojected and azimuthally averaged radial intensity profile of the continuum emission of LkCa 15 (left panel) and J1610 (right panel) as in Fig. 2. The blue ribbon shows the quadratic sum of the standard deviation of the intensity across pixels in each radial bin and the rms of the observations divided by the square root of the number of independent beams sampling the same radial bin in the azimuthal direction.
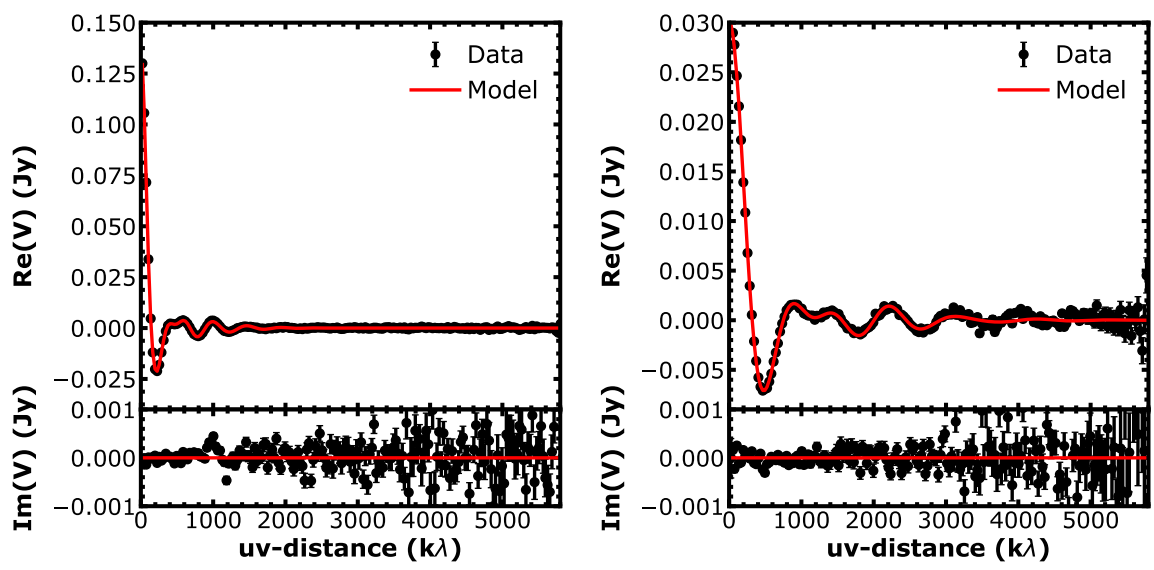

Fig. B.2. Re-centered and deprojected visibililities of the data and best fit model for LkCa 15 (left panel) and J1610 (right panel). Error bars show $1 \sigma$ uncertainties. The parameters of the best fits are listed in Tables 2 and 3. The plots have been made with the uvplot package (Tazzari 2017). 


\section{Appendix C: Initial disk temperature profile from MCRT iterations}

Figure C.1 shows the disk temperature profile obtained from the MCRT iterations described in Sect. 4.1 and the corresponding disk aspect ratio profile.
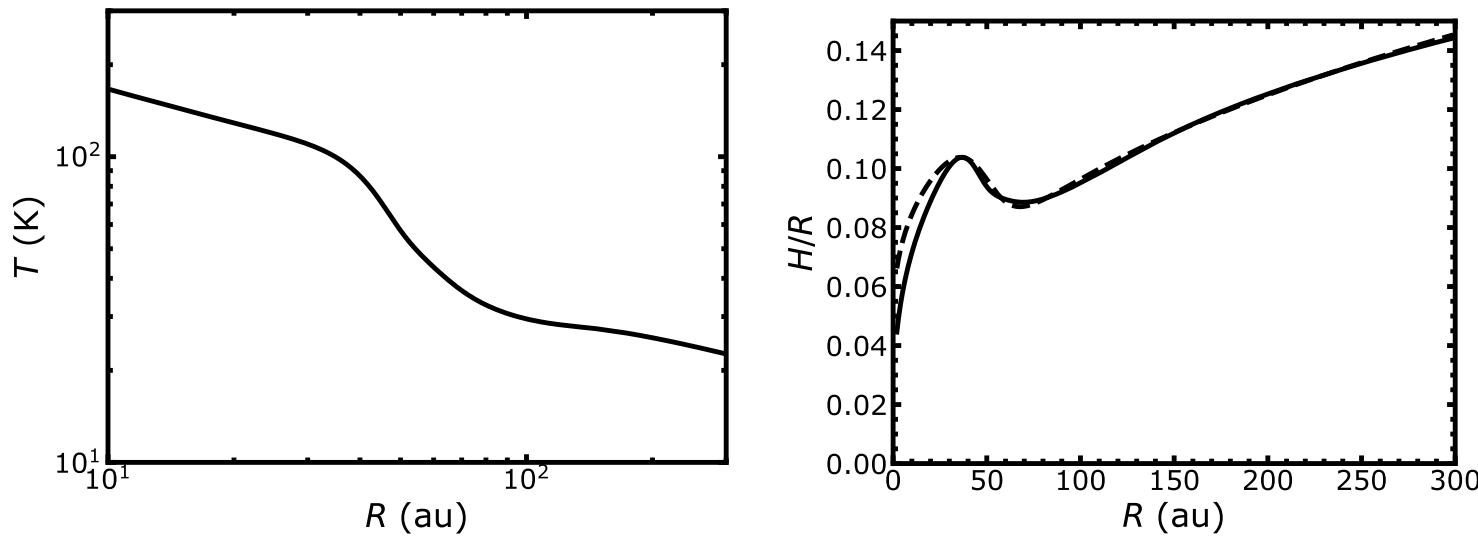

Fig. C.1. Left: density-weighted, vertically integrated disk temperature profile from the MCRT iterations described in Sect. 4.1. Right: disk aspect ratio $H / R$ corresponding to the temperature profile in the left panel, adopting a stellar mass of $1.25 M_{\odot}$ and a mean molecular weight of 2.4 . The dashed curve shows a fit to the profile between 30 and 300 au, as expressed by Eq. (3).

\section{Appendix D: On the point source within the LkCa 15 cavity}

Figure D.1, left, shows an image of LkCa 15 with color code stretched to highlight the point source like emission within the cavity. We imaged the disk by combining the short spacings observation with only one long baseline observations at the time. Interestingly, the point source like feature is clearly imaged when only the C43-9 observation is considered, but it is not apparent in the C43-8 plus short spacing image. This supports the discussion presented in Sect. 3.1, i.e. that the feature is an image artifact driven in particular by the C43-9 execution block. The right panel of Fig. D.1 shows the peak intensity within the area highlighted in the left panel, and how it varies with imaging parameters.
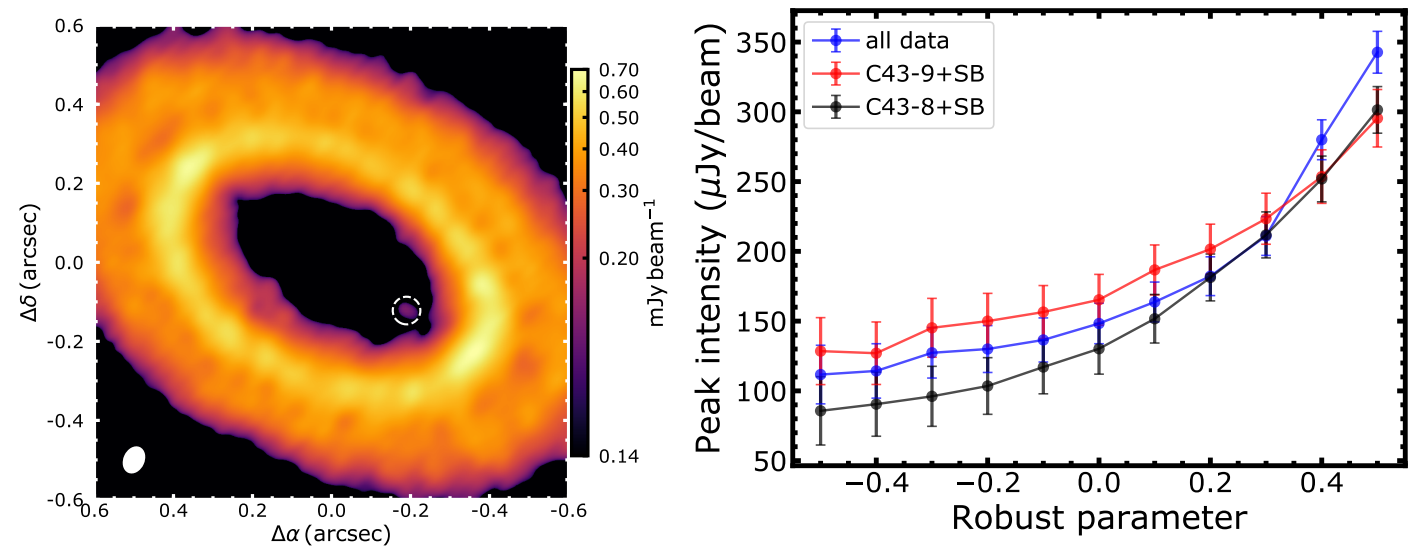

Fig. D.1. Left: zoom onto the cavity of LkCa 15, with stretched color scale to emphasize the fainter emission within the cavity. The white dashed circle indicates the presence of a potential point source within the cavity. Right: peak intensity of the potential point source in images generated with Briggs weighting but different robust parameters. The angular resolution is decreasing going from the left to the right of the plot. Different colors show the same analysis performed on the full data set, or on datasets including the short baselines and one of the two long baselines execution blocks, with C43-9 being the execution block of July 13th, and C43-8 being the execution block of July 19th (labeled following the nominal configurations of the observations.) 